\title{
A COMPARISON OF VARIOUS DEFINITIONS OF CONTRACTIVE MAPPINGS
}

BY

B. E. RHOADES

\begin{abstract}
A number of authors have defined contractive type mappings on a complete metric space $X$ which are generalizations of the well-known Banach contraction, and which have the property that each such mapping has a unique fixed point. The fixed point can always be found by using Picard iteration, beginning with some initial choice $x_{0} \in X$. In this paper we compare this multitude of definitions.

$X$ denotes a complete metric space with distance function $d$, and $f$ a function mapping $X$ into itself.
\end{abstract}

\section{Definitions of contractive type mappings.}

(1) (Banach) There exists a number $a, 0 \leqslant a<1$, such that, for each $x, y \in X$,

$$
d(f(x), f(y))<a d(x, y) .
$$

(2) (Rakotch [21]) There exists a monotone decreasing function $\alpha:(0, \infty)$ $\rightarrow[0,1)$ such that, for each $x, y \in X, x \neq y$,

$$
d(f(x), f(y)) \leqslant \alpha d(x, y) .
$$

(3) (Edelstein [10]) For each $x, y \in X, x \neq y$,

$$
d(f(x), f(y))<d(x, y) .
$$

(4) (Kannan [18]) There exists a number $a, 0<a<\frac{1}{2}$, such that, for each $x, y \in X$,

$$
d(f(x), f(y)) \leqslant a[d(x, f(x))+d(y, f(y))] .
$$

(5) (Bianchini [3]) There exists a number $h, 0 \leqslant h<1$, such that, for each $x, y \in X$,

$$
d(f(x), f(y)) \leqslant h \max \{d(x, f(x)), d(y, f(y))\} .
$$

(6) For each $x, y \in X, x \neq y$,

Received by the editors June 4, 1974 and, in revised form, March 5, 1975 and November 18, 1975.

AMS (MOS) subject classifications (1970). Primary 47H10.

- American Mathematical Society 1977 


$$
d(f(x), f(y))<\max \{d(x, f(x)), d(y, f(y))\} .
$$

(7) (Reich [24]) There exist nonnegative numbers $a, b, c$ satisfying $a+b+c$ $<1$ such that, for each $x, y \in X$,

$$
d(f(x), f(y)) \leqslant a d(x, f(x))+b d(y, f(y))+c d(x, y) .
$$

(8) (Reich [25]) There exist monotonically decreasing functions $a, b, c$ from $(0, \infty)$ into $[0,1)$ satisfying $a(t)+b(t)+c(t)<1$ such that, for each $x, y \in X$, $x \neq y$,

$$
\begin{aligned}
d(f(x), f(y)) \leqslant & a(d(x, y)) d(x, f(x)) \\
& +b(d(x, y)) d(y, f(y))+c(d(x, y)) d(x, y) .
\end{aligned}
$$

(9) There exist nonnegative functions $a, b, c$ satisfying

$$
\sup _{x, y \in X}\{a(x, y)+b(x, y)+c(x, y)\}<\lambda<1
$$

such that, for each $x, y \in X$,

$$
\begin{aligned}
d(f(x), f(y)) \leqslant & a(x, y) d(x, f(x)) \\
& +b(x, y) d(y, f(y))+c(x, y) d(x, y) .
\end{aligned}
$$

(10) (Sehgal [31]) For each $x, y \in X, x \neq y$,

$$
d(f(x), f(y))<\max \{d(x, f(x)), d(y, f(y)), d(x, y)\} .
$$

(11) (Chatterjea [5]) There exists a number $a, 0<a<\frac{1}{2}$, such that, for each $x, y \in X$,

$$
d(f(x), f(y)) \leqslant a\{d(x, f(y))+d(y, f(x))\} .
$$

(12) There exists a number $h, 0 \leqslant h<1$, such that, for each $x, y \in X$,

$$
d(f(x), f(y)) \leqslant h \max \{d(x, f(y)), d(y, f(x))\} .
$$

(13) For each $x, y \in X, x \neq y$,

$$
d(f(x), f(y))<\max \{d(x, f(y)), d(y, f(x))\} .
$$

(14) There exist nonnegative numbers $a, b, c$ satisfying $a+b+c<1$ such that, for each $x, y \in X$,

$$
d(f(x), f(y)) \leqslant a d(x, f(y))+b d(y, f(x))+c d(x, y) .
$$

(15) There exist monotone decreasing functions $a, b, c$ from $(0, \infty)$ into $[0,1)$ satisfying $a(t)+b(t)+c(t)<1$ such that, for each $x, y \in X, x \neq y$, 


$$
\begin{aligned}
d(f(x), f(y)) \leqslant & a(d(x, y)) d(x, f(y)) \\
& +b(d(x, y)) d(y, f(x))+c(d(x, y)) d(x, y) .
\end{aligned}
$$

(16) There exist nonnegative functions $a, b, c$ satisfying

$$
\sup _{x, y \in X}\{a(x, y)+b(x, y)+c(x, y)\} \leqslant \lambda<1
$$

such that, for each $x, y \in X$,

$$
\begin{aligned}
d(f(x), f(y)) \leqslant & a(x, y) d(x, f(y)) \\
& +b(x, y) d(y, f(x))+c(x, y) d(x, y) .
\end{aligned}
$$

(17) For each $x, y \in X, x \neq y$,

$$
d(f(x), f(y))<\max \{d(x, f(y)), d(y, f(x)), d(x, y)\} .
$$

(18) (Hardy and Rogers [15]) There exist nonnegative constants $a_{i}$ satisfying $\sum_{i=1}^{5} a_{i}<1$ such that, for each $x, y \in X$,

$$
\begin{aligned}
d(f(x), f(y)) \leqslant & a_{1} d(x, y)+a_{2} d(x, f(x))+a_{3} d(y, f(y)) \\
& +a_{4} d(x, f(y))+a_{5} d(y, f(x)) .
\end{aligned}
$$

(19) (Zamfirescu [37]) There exist real numbers $\alpha, \beta, \gamma, 0 \leqslant \alpha<1,0 \leqslant \beta$, $\gamma<\frac{1}{2}$, such that, for each $x, y \in X$, at least one of the following is true:

(i) $d(f(x), f(y)) \leqslant \alpha d(x, y)$,

(ii) $d(f(x), f(y)) \leqslant \beta[d(x, f(x))+d(y, f(y))]$,

(iii) $d(f(x), f(y)) \leqslant \gamma[d(x, f(y))+d(y, f(x))]$.

(20) For each $x, y \in X, x \neq y$,

$$
\begin{array}{r}
d(f(x), f(y))<\max \{d(x, y),[d(x, f(x))+d(y, f(y))] / 2, \\
[d(x, f(y))+d(y, f(x))] / 2\} .
\end{array}
$$

(21) (Ciric [7]) There exist nonnegative functions $q, r, s, t$ satisfying

$$
\sup _{x, y \in X}\{q(x, y)+r(x, y)+s(x, y)+2 t(x, y)\} \leqslant \lambda<1
$$

such that, for each $x, y \in X$,

$$
\begin{aligned}
d(f(x), f(y)) \leqslant & q(x, y) d(x, y)+r(x, y) d(x, f(x))+s(x, y) d(y, f(y)) \\
& +t(x, y)[d(x, f(y))+d(y, f(x))] .
\end{aligned}
$$


(22) For each $x, y \in X, x \neq y$,

$$
\begin{aligned}
d(f(x), f(y))<\max \{d(x, y), d(x, f(x)), d(y, f(y)), \\
{[d(x, f(y))+d(y, f(x))] / 2\} . }
\end{aligned}
$$

(23) There exist monotonically decreasing functions $\alpha_{i}:(0, \infty) \rightarrow[0,1)$ satisfying $\sum_{i=1}^{5} \alpha_{i}(t)<1$ such that, for each $x, y \in X, x \neq y$,

$$
\begin{aligned}
d(f(x), f(y)) \leqslant & \alpha_{1}(d(x, y)) d(x, f(x))+\alpha_{2}(d(x, y)) d(y, f(y)) \\
& +\alpha_{3}(d(x, y)) d(x, f(y))+\alpha_{4}(d(x, y)) d(y, f(x)) \\
& +\alpha_{5}(d(x, y)) d(x, y) .
\end{aligned}
$$

(24) (Ciric [8]) There exists a constant $h, 0 \leqslant h<1$, such that, for each $x, y \in X$,

$$
\begin{array}{r}
d(f(x), f(y)) \leqslant h \max \{d(x, y), d(x, f(x)), \\
\quad d(y, f(y)), d(x, f(y)), d(y, f(x))\} .
\end{array}
$$

(25) For each $x, y \in X, x \neq y$,

$$
\begin{aligned}
d(f(x), f(y))<\max \{d(x, y), d(x, f(x)), \\
\quad d(y, f(y)), d(x, f(y)), d(y, f(x))\} .
\end{aligned}
$$

We observe that the definition in Roux and Sardi [28] is a special case of (9), and that of Massa [19] is a special case of (19).

There exist functions $f$ with the property that some iterate of $f$ satisfies one of the preceding definitions, thereby giving rise to an additional twenty-five definitions, which we shall number 26-50.

For example,

(29) (Singh [32]) There exists a positive integer $p$ and a number $a, 0<a$ $<\frac{1}{2}$, such that, for each $x, y \in X$,

$$
d\left(f^{p}(x), f^{p}(y)\right) \leqslant a\left[d\left(x, f^{p}(x)\right)+d\left(y, f^{p}(y)\right)\right] .
$$

Chatterjea [5, p. 729] is (36); (48) and (49) appear in Ciric [7, p. 23] and [8, p. 271].

Let $p, q$ be fixed positive integers. We shall use 51-75 to identify those functions with the property that $f^{p}(x)$ and $f^{q}(y)$ satisfy one of the contractive conditions.

For example,

(51) (Yen [35]) There exist positive integers $p, q$ and a number $\alpha, 0<\alpha$ $<1$, such that, for each $x, y \in X$, 


$$
d\left(f^{p}(x), f^{q}(y)\right) \leqslant \alpha d(x, y) .
$$

Gupta and Srivistava [12, p. 94] is (64) with $c=0$.

It may happen that the particular iterate of $f$ depends on the point in the space. These are Definitions 76-100.

For example,

(76) (Guseman [14]) There exists a number $\alpha, 0<\alpha<1$, such that for each $x \in X$ there exists an integer $p(x)$, such that for each $y \in X$,

$$
d\left(f^{p(x)}(x), f^{p(x)}(y)\right) \leqslant \alpha d(x, y) .
$$

The iterate of $f$ may depend on both $x$ and $y$, giving us definitions 101-125. For example,

(103) (Bailey [1]) For each $x, y \in X, 0<d(x, y)$, implies there exists an integer $p=p(x, y)$ such that

$$
d\left(f^{p}(x), f^{p}(y)\right)<d(x, y) .
$$

2. Comparison of the definitions. Some of the above contractions, as originally defined by their respective authors, have additional hypotheses on $f$, such as continuity, and structure properties on $X$ such as compactness or uniform convexity. We shall make no such restrictions at this point. At the end of this section we shall impose additional restrictions as needed in order to establish the existence of a fixed point.

A statement like (a) $\Rightarrow(b)$ means that any function which satisfies condition (a) also satisfies condition (b).

From the way in which the definitions have been listed, it is obvious that, for $1 \leqslant m \leqslant 25$,

$$
(m) \Rightarrow(25+m) \Rightarrow(50+m) \text { and }(25+m) \Rightarrow(75+m) \Rightarrow(100+m) \text {. }
$$

We shall first establish a partial ordering for definitions (1)-(25), thereby giving immediately the corresponding partial orderings for (26)-(50), (51)-(75), (76)-(100), and (101)-(125).

Theorem 1. (i) (1) $\Rightarrow(2) \Rightarrow(3) \Rightarrow(10) ;(2) \nRightarrow(1),(3) \nRightarrow(1)$, and (10) $\nRightarrow(3)$.

(ii) $(2) \Rightarrow(8) \Rightarrow(23) ;(8) \nRightarrow(2)$.

(iii) (4) and (n) are independent, $n=1,2,3$.

(iv) (4) $\Rightarrow(5) \Rightarrow(6) \Rightarrow(10)$, but not conversely.

(v) (4) $\Rightarrow$ (7) $\Rightarrow(8) \Rightarrow(10)$; (7) $\nRightarrow$ (4).

(vi) (7) $\Rightarrow(9) \Rightarrow(10)$; (10) $\nRightarrow(9)$.

(vii) (6) and (n) are independent, $n=7,8,9$.

(viii) (5) $\Rightarrow(9)$, but not conversely. 
(ix) $(n) \nRightarrow(5), n=7,8$.

(x) (11) $\Rightarrow(12) \Rightarrow(13) \Rightarrow(17)$, but not conversely.

(xi) (11) $\Rightarrow$ (14) $\Rightarrow$ (15) $\Rightarrow$ (17); (14) $\nRightarrow$ (11).

(xii) (14) $\Rightarrow(16) \Rightarrow(17) ;(17) \nRightarrow(16)$.

(xiii) (6) and (13) are independent.

(xiv) (1) $\Rightarrow(18),(4) \Rightarrow(18)$, and (11) $\Rightarrow(18)$, but not conversely.

(xv) (12) $\Rightarrow(16)$, but not conversely.

(xvi) (13) and (14) are independent.

(xvii) $(13) \nRightarrow(n)$, for $n=16,18-21,24$.

(xviii) (10) and (13) are independent.

(xix) $(10) \nRightarrow(n), n=11,12$.

(xx) $(10) \Rightarrow(25)$ and $(13) \Rightarrow(25)$, but not conversely.

$(\mathrm{xxi})(9) \Rightarrow(21)$ and $(6) \Rightarrow(22)$.

(xxii) (15) $\Rightarrow(23)$ and (16) $\Rightarrow(24)$.

(xxiii) (16) and (21) are independent.

(xxiv) (17) $\Rightarrow(25)$, but not conversely.

(xxv) $(18) \Rightarrow(19) \Rightarrow(20) \Rightarrow(22) \Rightarrow(25) ;(20) \nRightarrow(19)$ and $(25) \nRightarrow(22)$.

$(\mathrm{xxvi})(19) \Rightarrow(21) \Rightarrow(24) \Rightarrow(25) ;(24) \nRightarrow(21)$ and $(25) \nRightarrow(24)$.

(xxvii) $(18) \Rightarrow(23) \Rightarrow(25)$.

(xxviii) $(21) \Rightarrow(22)$, but not conversely.

(xxix) $(m) \Rightarrow(25+m)$, but not conversely, $1<m \leqslant 25$.

(xxx) $(25+m) \Rightarrow(75+m)$, but not conversely, $1 \leqslant m<25$.

Most of the implications are obvious from the definitions. The nonobvious ones will be proved.

(i) The example on p. 8 of [10] shows that $(3) \nRightarrow(1)$.

To show that $(2) \neq(1)$, define $\alpha:(0, \infty) \rightarrow[0,1)$ by $\alpha(d)=1 /(d+1)$. Let $f(x)=1 /(x+1), 0 \leqslant x \leqslant 1$. Then $f:[0,1] \rightarrow[0,1]$ and has a fixed point at $(\sqrt{ } 5-1) / 2$. For any fixed $a, 0<a<1$, choose $y<-1+1 / a$ and $y \in(0,(\sqrt{ } 5-1) / 2)$. Then

$$
d(f(0), f(y))=y /(y+1)>a y=a d(0, y),
$$

and does not satisfy (1).

On the other hand, for $0 \leqslant x<y \leqslant 1$,

$$
d(f(x), f(y))=\frac{y-x}{(x+1)(y+1)}<\frac{y-x}{y-x+1}=\alpha(d) d(x, y),
$$

and $f$ satisfies (2).

Example 2, p. 574 of $[31]$ shows that $(10) \nRightarrow(3)$.

(ii) Let $f(x)=x / 3,0 \leqslant x<1, f(1)=\frac{1}{6}$. Then $f$ satisfies (8) but not (2).

(iii) Define $f(x)=\frac{1}{2}, 0 \leqslant x<1, f(1)=\frac{1}{4} . f \in(4)$, but $d(f(x), f(1))=\frac{1}{4}$ 
$>d(x, 1)$ for any $x \in\left(\frac{3}{4}, 1\right)$, so that $f$ does not satisfy (1)-(3).

With $f(x)=x / 3,0 \leqslant x \leqslant 1, f \in(1)$, hence (2) and (3), but $f \notin(4)$.

(iv) $f(x)=x / 3,0<x \leqslant 1$, satisfies (5) but not (4).

Let $f(x)=x^{2} / 2(x+1), 0 \leqslant x<\infty$. For $0<x<y$,

$$
\begin{aligned}
d(f(x), f(y)) & =(x y+x+y)(y-x) / 2(x+1)(y+1)<y(y+2) / 2(y+1) \\
& =\max \{d(x, f(x)), d(y, f(y))\},
\end{aligned}
$$

so that $f \in(6)$. However, $d(f(0), f(y))=y^{2} / 2(y+1)$. For any fixed $h$, $0<h<1$, one can find values of $y$ large enough so that $y /(y+2)>h$, and $f \notin(5)$.

To show that $(10) \neq(6)$ use Example 2 of [31, p. 574]; i.e., $f(x)=x / 2$, $0<x<4, f(x)=-2 x+10,4<x<5$. For $0<y \leqslant 4$,

$$
d(f(0), f(y))=y / 2=\max \{d(0, f(0)), d(y, f(y))\},
$$

and $f \notin(6)$.

(v) The example on p. 122 of [24] shows that $(7) \nRightarrow(4)$.

(vi) We note that (9) is equivalent to the following:

(9') There exists a constant $h, 0<h<1$, such that for each $x, y \in X$,

$$
d(f(x), f(y)) \leqslant h \max \{d(x, f(x)), d(y, f(y)), d(x, y)\} .
$$

To show that $(9) \Rightarrow\left(9^{\prime}\right)$, let

$$
M(x, y)=\max \{d(x, f(x)), d(y, f(y)), d(x, y)\} .
$$

Suppose $f \in(9)$. Then

$$
\begin{aligned}
d(f(x), f(y)) & \leqslant a(x, y) d(x, f(x)+b(x, y) d(y, f(y))+c(x, y) d(x, y) \\
& \leqslant(a(x, y)+b(x, y)+c(x, y)) M(x, y) \leqslant \lambda M(x, y),
\end{aligned}
$$

and $f$ satisfies $\left(9^{\prime}\right)$ with $h=\lambda$.

Conversely, suppose $f \in\left(9^{\prime}\right)$. At each point where $M(x, y)=d(x, f(x))$, define $a(x, y)=h, b(x, y)=c(x, y)=0$. At each point where $M(x, y)=$ $d(y, f(y))$, define $b(x, y)=\lambda, a(x, y)=c(x, y)=0$, and if $M(x, y)=d(x, y)$, define $c(x, y)=\lambda, a(x, y)=b(x, y)=0$. Then $f \in(9)$.

To show that $(10) \nRightarrow(9)$, we shall show that $(10) \nRightarrow\left(9^{\prime}\right)$. Let $f(x)$ $=x^{2} /(x+1), 0 \leqslant x<\infty$. Then

$$
d(f(x), f(y))=(x y+x+y)(y-x) /(x+1)(y+1)<y-x
$$

and $f \in(10)$. However, $d(f(0), f(y))=y^{2}(y+1)^{-1}>h y$ for any fixed $h$ less than 1 , and $y$ large enough. 
(vii) To show that $(n) \neq(6), n=7,8,9$, choose $f(x)=x / 2,0<x<1$. Then $f \in(7)$, but

$$
d(f(0), f(y))=y / 2=\max \{d(0, f(0)), d(y, f(y))\} .
$$

To show that $(6) \nRightarrow(n)$, let $f(x)=\frac{1}{2}, 0 \leqslant x \leqslant \frac{1}{2}, f(x)=0, \frac{1}{2}<x<1, f$ $\in(6)$. For $\frac{1}{2}<x \leqslant 1, d\left(f\left(\frac{1}{2}, f(x)\right)=\frac{1}{2}\right.$. Let

$$
g(x)=a d\left(\frac{1}{2}, f\left(\frac{1}{2}\right)\right)+b d(x, f(x))+c d\left(\frac{1}{2}, x\right) \cdot=b x+c\left(x-\frac{1}{2}\right) .
$$

As $x \rightarrow \frac{1}{2}+, g(x) \rightarrow b / 2 \leqslant \lambda / 2<\frac{1}{2}$, and $f \notin(9)$.

(viii) Using an argument similar to that which established the equivalence between (9) and $\left(9^{\prime}\right)$ in (vi), one can show that any $f \in(5)$ has a representation of the form (9) with $c \equiv 0$.

Let $f(x)=x / 2,0 \leqslant x \leqslant 1$. Then $f \in(9)$, but

$$
d(f(0), f(y))=y / 2=\max \{d(0, f(0)), d(y, f(y))\}>h y / 2
$$

for any $0 \leqslant h<1$.

(ix) Let $n=7,8$. To show that $(n) \nRightarrow(5)$, use the example from (vii) that $(n) \nRightarrow(6)$.

(x) Let $f(x)=0,0 \leqslant x<1, f(1)=\frac{1}{2}$. Then $f \in(12)$, but

$$
d\left(f\left(\frac{1}{2}\right), f(1)\right)=\frac{1}{2}=\left[d\left(\frac{1}{2}, f(1)\right)+d\left(1, f\left(\frac{1}{2}\right)\right)\right] / 2,
$$

and $f \notin(11)$.

To show (13) $\neq(12)$, define $f$ by $f(x)=x^{2} /(x+1), x>0 . f \in(13)$. $d(f(x), f(2 x))=x^{2}(2 x+3) /(2 x+1)(x+1)$. For $x>1$,

$$
\max \left\{d(x, f(2 x)), a^{\prime}(2 x, f(2 x))\right\}=x(x+2) /(x+1) .
$$

Thus, given any $0<h<1$ there exist values of $x$ large enough so that $x(2 x+3) /(2 x+1)(x+2)>h$.

Let $f(x)=1-x / 2,0 \leqslant x \leqslant 1$. Then $f \in(17)$, but $f \notin(13)$ for $x=0, y$ $=1$.

(xi) To show (14) $\nRightarrow(11)$, use the $f$ in $(x)$ for which (12) $\nRightarrow(11)$. This $f \in(14)$.

(xii) Using the technique in (vi) that (9) and ( $\left(9^{\prime}\right)$ are equivalent, we can show that (16) is equivalent to

(16') There exists a constant $h, 0 \leqslant h<1$, such that, for all $x, y \in X$,

$$
d(f(x), f(y)) \leqslant h \max \{d(x, f(y)), d(y, f(x)), d(x, y)\} .
$$

To show that $(17) \nRightarrow(16)$ pick the $f$ in $(x)$ for which $(13) \nRightarrow(12)$. Then 
$f \in(17)$. But

$$
\max \{d(x, f(2 x)), d(2 x, f(x)), d(x, 2 x)\}=x(x+2) /(x+1),
$$

and for $x$ sufficiently large $f \notin\left(16^{\prime}\right)$.

(xiii) Let $f(x)=\alpha(1-x), \frac{1}{2}<\alpha<1,0<x \leqslant 1$. Then $f \in(6)$, but $f$ does not satisfy (13) for $x=0, y=1$.

The $f$ in $(x)$ for which (17) $\nRightarrow(13)$ satisfies (6).

(xiv) To show that (18) $\nRightarrow(1)$, pick the $f$ in (iii) for which (4) $\nRightarrow(1)$.

To show that $(18) \nRightarrow(4)$, pick the $f$ in $(v)$ for which $(7) \nRightarrow(4)$. For $(18) \neq$ (11), use the $f$ in $(x)$ for which (12) $\nRightarrow(11)$.

(xv) The $f$ in (x) for which (17) $\nRightarrow(13)$ also satisfies (16).

(xvi) To show that $(13) \nRightarrow(14)$, pick the $f$ in (xii) for which (17) $\nRightarrow(16)$. That $f$ satisfies (13), but not (16), hence not (14).

From (xiii), the $f$ in $(x)$ for which $(17) \nRightarrow(13)$, satisfies (14).

(xvii) Using (xxv) and (xxvi), it will be sufficient to show that $(13) \nRightarrow(16)$, (13) $\Rightarrow(22)$, and (13) $\nRightarrow(24)$. Use the $f$ in $(x)$ for which (13) $\Rightarrow(12)$. From (xi), that $f$ does not satisfy (16).

$$
\begin{gathered}
\max \{d(x, 2 x), d(x, f(x)), d(2 x, f(2 x)), d(x, f(2 x)), d(2 x, f(x))\} \\
=x(x+2) /(x+1)
\end{gathered}
$$

so that, for $x$ large enough, $f \notin(24)$. For (13) $\nRightarrow(22)$, use the $f$ in (xviii) which satisfies (13) but not (10).

(xviii) To show that $(10) \nRightarrow(13)$, choose the $f$ in (xiii) for which (6) $\nRightarrow(13)$. This $f$ satisfies (10).

Let $f(x)=0,0 \leqslant x \leqslant \frac{1}{2}, f(x)=\frac{1}{2}, \frac{1}{2}<x \leqslant 1$. Then $f \in(13)$, but $f$ does not satisfy (10) for $x=\frac{1}{2}, y=1$.

(xix) From ( $x)$ it is sufficient to show that $(10) \nRightarrow(13)$, which follows from (xviii).

(xx) Let $f(x)=0,0 \leqslant x \leqslant \frac{1}{2}, f(x)=\frac{1}{2}, \frac{1}{2}<x \leqslant 1$. Then $f$ satisfies (25) but not (10). That $(25) \nRightarrow(13)$ follows from (xviii).

(xxiii) Let $f(x)=\varepsilon, 0 \leqslant x \leqslant \frac{1}{2}, f(x)=0, \frac{1}{2}<x \leqslant 1$, where $\frac{1}{4}<\varepsilon<\frac{1}{2}$. Then $f$ does not satisfy (16) for $x=\varepsilon, y=2 \varepsilon$.

Let $f(x)=0,0<x<1, f(1)=\frac{1}{2}$. Then $f \in(16)$, but $f$ does not satisfy (21) for $x=\frac{1}{2}, y=1$.

(xxiv) Use $f(x)=\frac{1}{2}, 0 \leqslant x \leqslant \frac{1}{2}, f(x)=0, \frac{1}{2}<x \leqslant 1$. $f$ does not satisfy (17) for $x=\frac{1}{2}, y=1$, but $f \in(22)$.

(xxv) In [15, p. 202] it is shown that (18) implies

(18') There exist nonnegative constants $a, b, c$ satisfying $a+2 b+2 c<1$ such that, for each $x, y \in X$, 


$$
\begin{aligned}
d(f(x), f(y)) \leqslant & a d(x, y)+b[c(x, f(x))+d(y, f(y))] \\
& +c[d(x, f(y))+d(y, f(x))] .
\end{aligned}
$$

It is obvious that $\left(18^{\prime}\right) \Rightarrow(18)$.

We shall now show that (19) is equivalent to

(19') There exist nonnegative functions $a, b, c$ satisfying

$$
\sup _{x, y \in X}\{a(x, y)+2 b(x, y)+2 c(x, y)\}<\lambda<1
$$

such that, for each $x, y \in X$,

$$
\begin{aligned}
d(f(x), f(y))< & a(x, y) d(x, y)+b(x, y)[d(x, f(x))+d(y, f(y))] \\
& +c(x, y)[d(x, f(y))+d(y, f(x))]
\end{aligned}
$$

and

(19") There exists a constant $h, 0<h<1$, such that for each $x, y \in X$,

$$
\begin{array}{r}
d(f(x), f(y)) \leqslant h \max \{d(x, y),[d(x, f(x))+d(y, f(y))] / 2, \\
[d(x, f(y))+d(y, f(x))] / 2\} .
\end{array}
$$

$(19) \Rightarrow\left(19^{\prime}\right)$. At each pair $x, y$ for which $f$ satisfies (19)(i), define $a(x, y)=\alpha, b=c=0$. At each pair $x, y$ for which $f$ satisfies (19)(ii), define $b(x, y)=\beta, a=c=0$, and similarly for (iii).

$\left(19^{\prime}\right) \Rightarrow\left(19^{\prime \prime}\right)$. Let

$$
\begin{aligned}
M(x, y)=\max \{d(x, y),[d(x, f(x))+d(y, f(y))] / 2, & \\
& {[d(x, f(y))+d(y, f(x))] / 2\} . }
\end{aligned}
$$

Let $f \in\left(19^{\prime}\right)$. Then

$$
d(f(x), f(y)) \leqslant[a(x, y)+2 b(x, y)+2 c(x, y)] M(x, y)<\lambda M(x, y),
$$

and $f \in\left(19^{\prime \prime}\right)$.

$\left(19^{\prime \prime}\right) \Rightarrow(19)$. At each pair $x, y$ for which $M(x, y)=d(x, y), f$ satisfies (19)(i) with $\alpha=h$. Whenever $M(x, y)=[d(x, f(x))+d(y, f(y))] / 2, f$ satisfies (19)(ii) with $\beta=h / 2$, and $f$ satisfies (19)(iii) with $\gamma=h / 2$ whenever $M(x, y)$ $=[d(x, f(y))+d(y, f(x))] / 2$.

(20) $\nRightarrow(19)$. Let $f(x)=x^{2} /(x+1), x \geqslant 0$. Then $d(f(x), f(y))<d(x, y)$ and $f \in(20)$. For $x \geqslant 1, d(f(x), f(2 x))=x^{2}(2 x+3) /(x+1)(2 x+1)$, and $\max \{d(x, 2 x),[d(x, f(x))+d(2 x, f(2 x))] / 2,[d(x, f(2 x))+d(2 x, f(x))] / 2\}=x$. 
Given any $h$ satisfying $0<h<1$, one can find values of $x$ large enough so that $x(2 x+3) /(x+1)(2 x+1)>h$, and $f \notin\left(19^{\prime \prime}\right)$.

$(25) \nRightarrow(22)$. Use the $f$ of $(x x)$.

(xxvi) Using an argument similar to that in (xxv), one can show that (21) is equivalent to

(21') There exists a constant $h, 0 \leqslant h<1$, such that for each $x, y \in X$,

$$
\begin{aligned}
d(f(x), f(y)) \leqslant h \max \{d(x, y), & d(x, f(x)), d(y, f(y)), \\
& {[d(x, f(y))+d(y, f(x))] / 2\}, }
\end{aligned}
$$

and

(21") (Zamfirescu [36]) There exists a constant $\alpha, 0 \leqslant \alpha<1$, such that for each distinct $x, y \in X$, at least one of the following holds:

(i) $d(f(x), f(y)) \leqslant \alpha d(x, y)$,

(ii) $d(f(x), f(y)) \leqslant \alpha d(x, f(x))$,

(iii) $d(f(x), f(y)) \leqslant \alpha d(y, f(y))$,

(iv) $d(f(x), f(y)) \leqslant(\alpha / 2)[d(x, f(y))+d(y, f(x))]$.

$(24) \nRightarrow(21)$. Use the $f$ of $(x)$ for which (12) $\nRightarrow(11)$. Then $f \in$ (24), but $f \notin(21)$.

$(25) \nRightarrow(24)$. Use the $f$ of $(\mathrm{xx})$.

(xxviii) Let $f(x)=\frac{1}{2}, 0 \leqslant x \leqslant \frac{1}{2}, f(x)=0, \frac{1}{2}<x \leqslant 1$. Then $f \in$ (22), but $f$ does not satisfy (21) for $x=\frac{1}{2}, y>\frac{1}{2}$ and near $\frac{1}{2}$.

(xxix) The inclusions are obvious. To show that $(25+m) \neq(m)$ for $1 \leqslant m \leqslant 25$, let $f(x)=0,0 \leqslant x \leqslant 1, x \neq \frac{1}{2}, f\left(\frac{1}{2}\right)=1$. Then $f \notin(25)$, hence $f \notin(m)$ for $1 \leqslant m<25$, but $f^{2} \equiv 0$ so that $f \in(n)$ for all $n>25$.

(xxx) To show that $(75+m) \nRightarrow(25+m)$, use the $f$ in the example on p. 105 of [1]. $f$ does not satisfy (50), since, for each $n$, one can choose $x$ $=(1 / n+1,0), y=(1 / n, 0)$. It is possible to show that, for each $x$ one can choose an integer $p(x)$ such that $f \in(76),(80)$, and (97), hence $f \in(75+m)$, $1 \leqslant m \leqslant 25$.

Although Theorem 1 has not exhausted all of the possible comparisons, it is complete enough to demonstrate that Definitions (124) and (125) are the most general.

It is interesting to note that, for each of the 125 definitions, if $f$ has a fixed point, it is unique. We shall demonstrate this fact for Definitions (124) and (125).

LEMMA. Let $f$ satisfy (124) or (125). If $f$ has a fixed point, then the fixed point is unique.

Suppose $u$ and $v$ are fixed points of $f, u \neq v$. Then, from (124), there exists an integer $p=p(u, v)$ such that 


$$
\begin{aligned}
d(u, v) & =d\left(f^{p}(u), f^{p}(v)\right) \\
& \leqslant h \max \left\{d(u, v), d\left(u, f^{p}(u)\right), d\left(v, f^{p}(v)\right), d\left(u, f^{p}(v)\right), d\left(v, f^{p}(u)\right)\right\} \\
& \leqslant h d(u, v),
\end{aligned}
$$

a contradiction. The corresponding proof for (125) is similar.

The following demonstrates that one must add continuity and the existence of a cluster point for $\left\{f^{n}\left(x_{0}\right)\right\}$ in order to ensure that $f$, in some of the definitions, possesses a fixed point.

THEOREM 2. Let $f$ satisfy any of the definitions (3), (10), (13), (17), (22), or (25) (or one of their analogues in (26)-(125)). Then the conditions (i) $f$ continuous and (ii) there exists a cluster point of $\left\{f^{n}\left(x_{0}\right)\right\}$ for some $x_{0} \in X$, are needed in order to ensure that every such $f$ possesses a fixed point.

Proof. As a result of (i), (iv), (x), (xxi), and (xxv) of Theorem 1, it is sufficient, for (i) of Theorem 2 , to construct functions satisfying (3), (6), or (13).

For (3), let $X=\left\{x_{n}=n \sqrt{ } 2+2^{n} \mid n=0, \pm 1, \pm 2, \ldots\right\}$, and define $f$ by $f\left(x_{n}\right)=x_{n-1}$. Then $f \in(3)$, but $f$ has no fixed point. (This example appears in [4].)

For (6), let $X=\left\{x_{n}=1-2^{-n-1} \mid n \geqslant 0\right\} \cup\{1\}, f(1)=\frac{1}{2}, f\left(x_{n}\right)=x_{n+1}$. Then $f \in(6)$, but $f$ has no fixed points.

For (13), let $X=[0, \infty), f(x)=[x]+1$. Then $f \in(13)$, but has no fixed points.

For (ii), let $X=[1, \infty), f(x)=x+x^{-1}$. Then $f \in(3)$ and (13). $f^{n}(x)$ is monotone increasing in $n$. Since $f^{k+1}(x)-f^{k}(x)=1 / f^{k}(x)$, we obtain

$$
f^{n+1}(x)-f(x)=\sum_{k=1}^{n} 1 / f^{k}(x)>n / f^{n}(x) .
$$

Replacing $f^{n+1}(x)$ with $f^{n}(x)+1 / f^{n}(x)$ yields

$$
n-1<\left(f^{n}(x)\right)^{2}\left[f^{n}(x)-f(x)\right]<\left(f^{n}(x)\right)^{3} .
$$

Thus $f^{n}(x)>(n-1)^{1 / 3}$ and $f$ has no fixed points.

If $f$ satisfies (6) then $\left\{f^{n}(x)\right\}$ is bounded for each $x \in X$. For, if $f^{N+1}(x)$ $=f(x)$ for some integer $N$, then $f^{n}(x)=f(x)$ for all $n \geqslant N$ and $\left\{f^{n}(x)\right\}$ is convergent, hence bounded. Assume $f^{m}(x) \neq f^{n}(x)$ for all $m, n \geqslant 0, m \neq n$. Then

$$
d\left(f^{n+1}(x), f^{n+2}(x)\right)<d\left(f^{n}(x), f^{n+1}(x)\right)<\cdots<d(x, f(x)),
$$

so that $d\left(f(x), f^{n}(x)\right)<d(x, f(x))$ and hence $\left\{f^{n}(x)\right\}$ is bounded. Thus, in any finite dimensional space $\left\{f^{n}(x)\right\}$ will automatically have a cluster point. 
Throughout the rest of the paper we shall assume that any $f$, satisfying any of the definitions referred to in Theorem 2, is continuous on $X$, and that there exists a cluster point of $\left\{f^{n}\left(x_{0}\right)\right\}$ for some $x_{0} \in X$.

3. Fixed point theorems. Since we do not have any fixed point theorem for definition (25), we shall list the best fixed point theorem we can obtain for the definitions in each of the strata (1)-(25), (26)-(50), etc.

THEOREM 3. Let $f$ be continuous and satisfy (22). If $z$ is a cluster point of $\left\{f^{n}\left(x_{0}\right)\right\}$ for some $x_{0} \in X$, then $z$ is the unique fixed point of $f$ and $f^{n}\left(x_{0}\right) \rightarrow z$.

Theorem 3 is the special case of Theorem 6 with $p=1$. Special cases of Theorem 3 appear in [10] and [31].

Theorem 4. Let $f \in(23), x_{0} \in X$. Then $f$ has a unique fixed point $z$ and $f^{n}\left(x_{0}\right) \rightarrow z$.

Proof. Define the sequence $\left\{x_{0}, x_{1}=f\left(x_{0}\right), \ldots, x_{n+1}=f\left(x_{n}\right), \ldots,\right\}$. Since $f \in(23)$,

$$
\begin{aligned}
d\left(x_{n}, x_{n+1}\right)= & d\left(f\left(x_{n-1}\right), f\left(x_{n}\right)\right) \\
\leqslant & \alpha_{1} d\left(x_{n-1}, x_{n}\right)+\alpha_{2} d\left(x_{n}, x_{n+1}\right) \\
& +\alpha_{3} d\left(x_{n-1}, x_{n+1}\right)+\alpha_{5} d\left(x_{n-1}, x_{n}\right),
\end{aligned}
$$

where, for brevity, we let $\alpha_{i}=\alpha_{i}\left(d\left(x_{n-1}, x_{n}\right)\right)$. Because of the symmetry in Definition (23),

$$
\begin{aligned}
d\left(x_{n+1}, x_{n}\right) \leqslant & \alpha_{1} d\left(x_{n}, x_{n+1}\right)+\alpha_{2} d\left(x_{n-1}, x_{n}\right) \\
& +\alpha_{4} d\left(x_{n-1}, x_{n+1}\right)+\alpha_{5} d\left(x_{n}, x_{n-1}\right) .
\end{aligned}
$$

Adding we have

$$
\begin{aligned}
2 d\left(x_{n}, x_{n+1}\right) \leqslant & \left(\alpha_{1}+\alpha_{2}+2 \alpha_{5}\right) d\left(x_{n-1}, x_{n}\right)+\left(\alpha_{2}+\alpha_{1}\right) d\left(x_{n}, x_{n+1}\right) \\
& +\left(\alpha_{3}+\alpha_{4}\right) d\left(x_{n-1}, x_{n+1}\right) .
\end{aligned}
$$

But $d\left(x_{n-1}, x_{n+1}\right) \leqslant d\left(x_{n-1}, x_{n}\right)+d\left(x_{n}, x_{n+1}\right)$, so that

$$
\begin{aligned}
d\left(x_{n}, x_{n+1}\right) & \leqslant\left(\alpha_{1}+\alpha_{2}+\alpha_{3}+\alpha_{4}+2 \alpha_{5}\right) d\left(x_{n-1}, x_{n}\right) /\left(2-\alpha_{1}-\alpha_{2}-\alpha_{3}-\alpha_{4}\right) \\
& <d\left(x_{n-1}, x_{n}\right),
\end{aligned}
$$

since $\sum_{i=1}^{5} \alpha_{i}(t)<1$.

Thus $\left\{d\left(x_{n}, x_{n+1}\right)\right\}$ is monotone decreasing in $n$. Call the limit $p$, and assume that $p>0$.

Let 


$$
q(t)=\frac{\alpha_{1}(t)+\alpha_{2}(t)+\alpha_{3}(t)+\alpha_{4}(t)+2 \alpha_{5}(t)}{2-\alpha_{1}(t)-\alpha_{2}(t)-\alpha_{3}(t)-\alpha_{4}(t)}
$$

Then $b_{n}=d\left(x_{n}, x_{n+1}\right) \leqslant p$ implies $q\left(b_{n}\right) \leqslant q(p)<1$, for all $n$, so that

$$
d\left(x_{n+1}, x_{n}\right) \leqslant q(p) d\left(x_{n}, x_{n-1}\right) \leqslant \cdots \leqslant(q(p))^{n} d\left(x_{1}, x_{0}\right) \rightarrow 0
$$

as $n \rightarrow \infty$.

We shall now show that $\left\{x_{n}\right\}$ is Cauchy. For each pair of integers $m, n$, and assuming that $d\left(x_{m-1}, x_{n-1}\right) \neq 0$,

$$
\begin{aligned}
d\left(x_{m}, x_{n}\right) \leqslant & \alpha_{1} d\left(x_{m-1}, x_{m}\right)+\alpha_{2} d\left(x_{n-1}, x_{n}\right)+\alpha_{3} d\left(x_{m-1}, x_{n}\right) \\
& +\alpha_{4} d\left(x_{n-1}, x_{m}\right)+\alpha_{5} d\left(x_{m-1}, x_{n-1}\right),
\end{aligned}
$$

which can be written in the form

$$
d\left(x_{m}, x_{n}\right) \leqslant \frac{\left(\alpha_{1}+\alpha_{3}+\alpha_{5}\right) d\left(x_{m-1}, x_{m}\right)+\left(\alpha_{2}+\alpha_{4}+\alpha_{5}\right) d\left(x_{n-1}, x_{n}\right)}{1-\alpha_{3}-\alpha_{4}-\alpha_{5}} .
$$

Let $r(t)=\beta(t) / \xi(t), s(t)=\gamma(t) / \xi(t)$, where $\beta(t)=\alpha_{1}(t)+\alpha_{3}(t)+\alpha_{5}(t)$, $\gamma(t)=\alpha_{2}(t)+\alpha_{4}(t)+\alpha_{5}(t)$, and $\xi(t)=1-\alpha_{3}(t)-\alpha_{4}(t)-\alpha_{5}(t)$. Note that $r$ and $s$ are monotone decreasing in $t$.

Fix $\varepsilon>0$. If $\beta(\varepsilon) \neq 0$ and $\gamma(\varepsilon) \neq 0$, there exists an $N$ such that, for $m, n \geqslant N$,

$$
d\left(x_{m-1}, x_{m}\right)<\frac{1}{2} \min \{\varepsilon / r(\varepsilon / 2), \varepsilon\}
$$

and $d\left(x_{n-1}, x_{n}\right)<\frac{1}{2} \min \{\varepsilon / s(\varepsilon / 2), \varepsilon\}$. If $r(\varepsilon)=0$, for example, then choose $N$ so that $m \geqslant N$ implies $d\left(x_{m-1}, x_{m}\right)<\varepsilon / 2$.

For each $m, n$ such that $d\left(x_{m-1}, x_{n-1}\right)>\varepsilon / 2$, it follows that

$$
d\left(x_{m}, x_{n}\right) \leqslant r(\varepsilon / 2) d\left(x_{m-1}, x_{m}\right)+s(\varepsilon / 2) d\left(x_{n-1}, x_{n}\right)<\varepsilon / 2+\varepsilon / 2=\varepsilon .
$$

For each $m, n$ such that $d\left(x_{m-1}, x_{n-1}\right)<\varepsilon / 2$, and again using the symmetry property of the $\alpha_{i}$ 's along with the triangular inequality, we can express

$$
\begin{aligned}
d\left(x_{m}, x_{n}\right) \leqslant & \frac{1}{2}\left(\alpha_{1}+\alpha_{2}+\alpha_{3}+\alpha_{4}\right)\left[d\left(x_{m-1}, x_{m}\right)+d\left(x_{n-1}, x_{n}\right)\right] \\
& +\left(\alpha_{3}+\alpha_{4}+\alpha_{5}\right) d\left(x_{m-1}, x_{n-1}\right) \\
< & \left(\alpha_{1}+\alpha_{2}+2 \alpha_{3}+2 \alpha_{4}+\alpha_{5}\right) \varepsilon / 2<\varepsilon .
\end{aligned}
$$

Let $z=\lim _{n} x_{n}$. It remains to show that $z$ is a fixed point of $f$. We shall first show that $x_{n+1} \rightarrow f(z)$. 
Assuming that $z \neq x_{n}$ for any $n, f \in(23)$ implies that

$$
\begin{aligned}
& d\left(x_{n+1}, f(z)\right) \\
& \quad \leqslant \frac{\left(\alpha_{1}+\alpha_{3}\right) d\left(x_{n}, x_{n+1}\right)+\left(\alpha_{2}+\alpha_{4}\right) d\left(z, x_{n+1}\right)+\alpha_{5} d\left(x_{n}, z\right)}{1-\alpha_{2}-\alpha_{3}} .
\end{aligned}
$$

From the symmetry of the $\alpha_{i}$ 's,

$$
\begin{aligned}
& d\left(f(z), x_{n+1}\right) \\
& \quad \leqslant \frac{\left(\alpha_{1}+\alpha_{3}\right) d\left(z, x_{n+1}\right)+\left(\alpha_{2}+\alpha_{4}\right) d\left(x_{n}, x_{n+1}\right)+\alpha_{5} d\left(z, x_{n}\right)}{1-\alpha_{1}-\alpha_{4}} .
\end{aligned}
$$

Since $\alpha_{2}+\alpha_{3}$ and $\alpha_{1}+\alpha_{4}$ are both evaluated at $d\left(x_{n}, z\right)$, and since $\sum_{i=1}^{5} \alpha_{i}(t)<1$ for each $t>0$, it follows that at least one of the sums, say $\alpha_{2}+\alpha_{3}$, must be less than $\frac{1}{2}$ for an infinite number of choices $n_{i}$ of $n$. Thus $\lim _{i} d\left(f(z), x_{n_{i}+1}\right)=0$. Since $d\left(x_{n}, x_{n+1}\right)$ is monotone decreasing in $n$, we can conclude that $x_{n+1} \rightarrow f(z)$. Since $x_{n} \rightarrow z, z=f(z)$. Uniqueness follows from the Lemma.

Special cases of Theorem 4 appear in [5], [10], [15], [18], [21], [24], [25], and [31].

TheORem 5. Let $f \in(24), x_{0} \in X$. Then $f$ has a unique fixed point $z$ and $f^{n}\left(x_{0}\right) \rightarrow z$.

Theorem 5, which appears in [8], is the special case of Theorem 8 for $p=1$. Special cases of Theorem 5 appear in [3], [5], [7], [15], [18], [24], and [37].

For definitions (26) $-(50)$ we have the following.

THEOREM 6. Let $f \in(47), f$ continuous. If $z$ is a cluster point of $\left\{f^{p n}\left(x_{0}\right)\right\}$ for some $x_{0} \in X$, then $z$ is the unique fixed point of $f$ and $f^{n}\left(x_{0}\right) \rightarrow z$.

Theorem 6 follows immediately from Theorem 9 by setting $q=p$.

THEOREM 7. Let $f \in(48), x_{0} \in X$. Then $f$ has a unique fixed point $z$ and $f^{n}\left(x_{0}\right) \rightarrow z$.

Proof. Let $x_{0} \in X$ and define the sequence $\left\{x_{n}\right\}$ by $x_{n+1}=f\left(x_{n}\right), n \geq 0$. Let $w$ be any fixed integer satisfying $0 \leqslant w<p$, and consider the subsequence $\left\{x_{w}, x_{w+p}, \ldots, x_{w+n p}, \ldots\right\}$. Employing the argument of Theorem 4 on this subsequence, with $f$ replaced by $f^{p}$, we conclude that $f^{n p}\left(x_{w}\right) \rightarrow z_{w}$ and $z_{w}$ is a fixed point of $f^{p}$. Let $y$ and $z$ denote the values of $z_{w}$ for two different choices of $w$. Suppose $y \neq z$. Then 


$$
\begin{aligned}
d(y, z)=d\left(f^{p}(y), f^{p}(z)\right) \leqslant & \alpha_{1} d\left(y, f^{p}(y)\right)+\alpha_{2} d\left(z, f^{p}(z)\right) \\
& +\alpha_{3} d\left(y, f^{p}(z)\right)+\alpha_{4} d\left(z, f^{p}(y)\right)+\alpha_{5} d(y, z) \\
= & \left(\alpha_{3}+\alpha_{4}+\alpha_{5}\right) d(y, z)<d(y, z),
\end{aligned}
$$

a contradiction. Therefore each of the subsequences $\left\{x_{w+n p}\right\}_{n=0}^{\infty}$ converges to the same limit. Call it $z$. Consequently $\left\{x_{n}\right\}$ converges to $z$, and $z$ is a fixed point of $f^{p} \cdot f^{p}(f(z))=f\left(f^{p}(z)\right)=f(z)$, so that $f(z)$ is also a fixed point of $f^{p}$. But the above argument shows that $f^{p}$ has a unique fixed point. Therefore $f(z)=z$ and $z$ is a fixed point of $f$. Uniqueness follows from the Lemma.

THEOREM 8. Let $f \in(49), x_{0} \in X$. Then $f$ has a unique fixed point $z$ and $f^{n}\left(x_{0}\right) \rightarrow z$.

Theorem 8 is the special case of Theorem 11 for which $p=q$.

THEOREM 9. Let $f$ be continuous and satisfy (72). If $z$ is a cluster point of $\left\{f^{(p+q) n}\left(x_{0}\right)\right\}$ for some $x_{0} \in X$, then $z$ is the unique fixed point of $f$ and $f^{n}\left(x_{0}\right) \rightarrow z$.

Proof. From Theorem 15 , replacing $f$ by $f^{p}$, and $g$ by $f^{q}$, we get that $z$ is the unique fixed point of $f$, and $f^{(p+q) n}\left(x_{0}\right) \rightarrow z$. For any $m>p+q, m$ $=r(p+q)+s$, where $r \geqslant 1,0 \leqslant s<p+q$.

Since $f$ is continuous,

$$
d\left(f^{m}\left(x_{0}\right), z\right)=d\left(f^{r(p+q)+s}\left(x_{0}\right), z\right) \rightarrow d\left(f^{s}(z), z\right)=0 .
$$

The presence of $f^{p}$ and $f^{q}$ for different values of $p$ and $q$, in Definitions (51)-(75) wipes out the symmetry relationships that held, for example, in (18) and (23). Consequently, Theorem 10 has stronger hypotheses and a weaker conclusion than Theorem 7.

THEOREM 10. Let $f \in(73)$, with the added restriction that the $\alpha_{i}$ satisfy

$$
r(t) \cdot s(t)=\frac{\alpha_{1}(t)+\alpha_{3}(t)+\alpha_{5}(t)}{1-\alpha_{2}(t)-\alpha_{3}(t)} \cdot \frac{\alpha_{2}(t)+\alpha_{4}(t)+\alpha_{5}(t)}{1-\alpha_{1}(t)-\alpha_{4}(t)}<1
$$

for each $t>0$. Let $x_{0} \in X$. Then either $f^{p}$ or $f^{q}$ has a fixed point. If, in addition

$$
\lim _{t \rightarrow 0+} \alpha_{2}(t)+\alpha_{3}(t)<1 \text { and } \lim _{t \rightarrow 0+} \alpha_{1}(t)+\alpha_{4}(t)<1,
$$

then $f$ has a unique fixed point $z$ and $f^{n}\left(x_{0}\right) \rightarrow z$.

Proof. Define the sequence $\left\{x_{0}, x_{1}=f\left(x_{0}\right), \ldots, x_{n+1}=f\left(x_{n}\right), \ldots\right\}$, and consider the subsequence $\left\{x_{w}, x_{q+w}, \ldots, x_{n(p+q)+w}, x_{n(p+q)+q+w}, \ldots\right\}$, where $w$ is any fixed integer satisfying $0 \leqslant w<p+q$. Assume $x_{n} \neq x_{m}$ for each $n \neq m$. Since $f \in(73)$, 


$$
\begin{aligned}
& d\left(x_{n(p+q)+w}, x_{n(p+q)+q+w}\right)=d\left(f^{p}\left(x_{(n-1)(p+q)+q+w}\right), f^{q}\left(x_{n(p+q)+w}\right)\right) \\
& <\alpha_{1} d\left(x_{(n-1)(p+q)+q+w}, x_{n(p+q)+w}\right)+\alpha_{2} d\left(x_{n(p+q)+w}, x_{n(p+q)+q+w}\right) \\
& \quad+\alpha_{3} d\left(x_{(n-1)(p+q)+q+w}, x_{q+n(p+q)+w}\right)+\alpha_{5} d\left(x_{(n-1)(p+q)+q+w}, x_{n(p+q)+w}\right) .
\end{aligned}
$$

Thus

$$
d\left(x_{n(p+q)+w}, x_{n(p+q)+q+w}\right) \leqslant r\left(b_{n}\right) d\left(x_{(n-1)(p+q)+q+w}, x_{n(p+q)+w}\right),
$$

where $b_{n}=d\left(x_{(n-1)(p+q)+w}, x_{n(p+q)+w}\right)$. Similarly,

$$
d\left(x_{(n-1)(p+q)+q+w}, x_{n(p+q)+w}\right) \leqslant s\left(b_{n}\right) d\left(x_{(n-1)(p+q)+w}, x_{(n-1)(p+q)+q+w}\right) .
$$

Combining these two inequalities and using condition (i), the sequence $\left\{c_{n}\right\}$, where $c_{n}=d\left(x_{n(p+q)+w}, x_{n(p+q)+q+w}\right)$ is monotone decreasing in $n$. Let $u$ denote the limit, and assume $u>0$.

Set $v=r(u) s(u)$. Then $c_{n} \geqslant u$ implies $r\left(c_{n}\right) s\left(c_{n}\right)<q$ for all $n$. Therefore $c_{n}<v c_{n-1}<\cdots<v^{n} c_{0} \rightarrow 0$.

Using the fact that $f \in(73)$, and the triangular inequality, we may write

$$
d\left(x_{n(p+q)+q+w}, x_{m(p+q)+w}\right)<\left[\beta\left(d_{m n}\right) b_{m}+\gamma\left(d_{m n}\right) c_{n}\right] / \xi\left(d_{m n}\right),
$$

where $d_{m n}=d\left(x_{(m-1)(p+q)+q+w}, x_{n(p+q)+w}\right)$, and $\beta, \gamma, \xi$ are defined as in the proof of Theorem 4.

Fix $\varepsilon>0$ and assume $d_{m n} \neq 0$ for each $m$ and $n$. Then, as in the proof of Theorem 4, we can find an integer such that $m, n>N$ imply $c_{n}<\varepsilon$ and $d\left(x_{n(p+q)+q+w}, x_{m(p+q)+w}\right)<\varepsilon$. Thus $d\left(x_{n(p+q)+w}, x_{m(p+q)+w}\right)<2 \varepsilon$.

$$
d\left(x_{n(p+q)+q+w}, x_{m(p+q)+q+w}\right) \leqslant d\left(x_{n(p+q)+q+w}, x_{m(p+q)+w}\right)+c_{m}<2 \varepsilon .
$$

Similarly, $d\left(x_{n(p+q)+w}, x_{m(p+q)+q+w}\right)<2 \varepsilon$. Therefore the sequence $\left\{x_{w}, x_{q+w}\right.$, $\left.x_{p+q+w}, \ldots\right\}$ is Cauchy, hence convergent. Call the limit $z_{w}$.

In a similar manner we can show that the sequence $\left\{x_{w}, x_{p+w}, x_{p+q+w}, \ldots\right.$, $\left.x_{n}(p+q)+w, x_{n}(p+q)+p+w, \ldots\right\}$ is Cauchy, hence convergent. Call the limit $x_{w}$. Note that the two sequences $\left\{x_{w}, x_{q+w}, x_{p+q+w}, \ldots\right\}$ and $\left\{x_{w}, x_{p+w}\right.$, $\left.x_{p+q+w}, \ldots\right\}$ have the subsequence $\left\{x_{n(p+q)+w}\right\}$ in common, so that $x_{w}=z_{w}$.

Using (73) and the triangular inequality we may write, provided $z_{w}$ $\neq x_{n(p+q)+w}$ for any $n$,

$$
\begin{aligned}
& d\left(f^{p}\left(z_{w}\right), f^{q}\left(x_{n(p+q)+w}\right)\right) \\
& \leqslant \frac{\left(\alpha_{1}+\alpha_{3}\right) d\left(z, x_{n(p+q)+q+w}\right)+\left(\alpha_{2}+\alpha_{4}\right) c_{n}+\alpha_{5} d\left(z_{w}, x_{n(p+q)+w}\right)}{1-\alpha_{1}-\alpha_{4}}
\end{aligned}
$$

and 


$$
\begin{aligned}
& d\left(f^{p}\left(x_{m(p+q)+w}, f^{q}\left(z_{w}\right)\right)\right. \\
& <\frac{\left(\alpha_{1}+\alpha_{3}\right) d\left(x_{m(p+q)+w}, x_{m(p+q)+p+w}\right)+\left(\alpha_{2}+\alpha_{4}\right) d\left(z_{w}, x_{m(p+q)+p+w}\right)+\alpha_{5} d\left(x_{m(p+q)+w}, z_{w}\right)}{1-\alpha_{2}-\alpha_{3}},
\end{aligned}
$$

where the $\alpha_{i}$ are evaluated at $d\left(z_{w}, x_{n(p+q)+w}\right)$. It then follows, as in Theorem 4 , that at least one of the sums $\alpha_{1}+\alpha_{4}$ or $\alpha_{2}+\alpha_{3}$ is less than or equal to $\frac{1}{2}$ for an infinite number of values $n_{i}$ of $n$.

Thus, from (251) and (252), either $x_{n(p+q)+q+w} \rightarrow f^{p}\left(z_{w}\right)$ or $x_{n(p+q)+p+w}$ $\rightarrow f^{q}\left(z_{w}\right)$, so that $z_{w}$ is a fixed point of either $f^{p}$ or $f^{q}$.

If condition (ii) is satisfied, then, from (251) and (252) both $f^{p}$ and $f^{q}$ have $z_{w}$ as a fixed point.

Let $y, z$ be two values of $z_{w}$ for different values of $w$, and assume $y \neq z$. Then

$$
\begin{aligned}
d(y, z)= & d\left(f^{p}(y), f^{q}(z)\right) \leqslant \\
& \alpha_{1} d\left(y, f^{p}(y)\right)+\alpha_{2} d\left(z, f^{q}(z)\right) \\
& +\alpha_{3} d\left(y, f^{q}(z)\right)+\alpha_{4} d\left(z, f^{p}(y)\right)+\alpha_{5} d(y, z) \\
= & \left(\alpha_{3}+\alpha_{4}+\alpha_{5}\right) d(y, z)<d(y, z),
\end{aligned}
$$

a contradiction. Therefore each of the subsequences of $\left\{x_{n}\right\}$ has the same limit, and $\left\{x_{n}\right\}$ converges to this limit. Call it $z$. Moreover, $z$ is the unique fixed point of $f^{p}$. Then $f^{p}(z)=z$, so that $f^{p}(f(z))=f(z)$, which implies $f(z)=z$. The uniqueness follows from the lemma.

Let $O(x, n)=\left\{x, f(x), \ldots, f^{n}(x)\right\}$. For any set $A$ let

$$
\delta(A)=\sup \{d(x, y) \mid x, y \in A\} .
$$

THEOREM 11. Let $f \in(74), x_{0} \in X$. Then $f$ has a unique fixed point $z$ and $f^{n}\left(x_{0}\right) \rightarrow z$.

Proof. Define the sequence $\left\{x_{0}, x_{1}=f\left(x_{0}\right), \ldots, x_{n+1}=f\left(x_{n}\right), \ldots\right\}$. Let $j$, $k$ be positive integers, $j>k \geqslant p+q$. Then there exist integers $m, n, r, s$ with $r, s$ satisfying $0 \leqslant r, s<p+q$, such that $j=m(p+q)+r$ and $k=$ $n(p+q)+s$. Since $f \in(74)$,

$$
\begin{aligned}
d\left(x_{j}, x_{k}\right) \mid \leqslant h \max \{ & d\left(x_{(m-1)(p+q)+q+r}, x_{(n-1)(p+q)+p+s}\right), \\
& d\left(x_{(m-1)(p+q)+q+r}, x_{m(p+q)+r}\right), \\
& d\left(x_{(n-1)(p+q)+p+s}, x_{n(p+q)+s}\right), \\
& d\left(x_{(m-1)(p+q)+q+r}, x_{n(p+q)+s}\right), \\
& \left.d\left(x_{(n-1)(p+q)+p+s}, x_{m(p+q)+r}\right)\right\},
\end{aligned}
$$


(254)

$d\left(x_{(m-1)(p+q)+q+r}, x_{(n-1)(p+q)+p+s}\right)$

$\leqslant h \max \left\{d\left(x_{(n-1)(p+q)+s}, x_{(m-1)(p+q)+r}\right)\right.$,

$d\left(x_{(n-1)(p+q)+s}, x_{(n-1)}(p+q)+p+s\right)$,

$d\left(x_{(m-1)}(p+q)+r, x_{(m-1)(p+q)+q+r)}\right.$

$d\left(x_{(n-1)}(p+q)+s, x_{(m-1)}(p+q)+q+r\right)$,

$\left.d\left(x_{(m-1)}(p+q)+r, x_{(n-1)(p+q)+p+s}\right)\right\}$,

$d\left(x_{(m-1)(p+q)+q+r}, x_{m(p+q)+r}\right)$

$\leqslant h \max \left\{d\left(x_{(m-1)(p+q)+q+r}, x_{(m-1)(p+q)+r}\right)\right.$,

(255)

$$
\begin{aligned}
& d\left(x_{(m-1)(p+q)+q+r}, x_{m(p+q)+r}\right) \\
& d\left(x_{(m-1)(p+q)+r}, x_{(m-1)(p+q)+q+r}\right) \\
& \left.d\left(x_{(m-1)(p+q)+r}, x_{m(p+q)+r}\right)\right\}
\end{aligned}
$$

$d\left(x_{(n-1)(p+q)+p+s}, x_{n(p+q)+s}\right)$

$\leqslant h \max \left\{d\left(x_{(n-1)}(p+q)+s, x_{(n-1)}(p+q)+p+s\right)\right.$,

(256)

$$
\begin{aligned}
& d\left(x_{(n-1)(p+q)+s}, x_{(n-1)}(p+q)+p+s\right), \\
& d\left(x_{(n-1)(p+q)+p+s}, x_{n(p+q)+s}\right), \\
& \left.\quad d\left(x_{(n-1)(p+q)+s}, x_{n(p+q)+s}\right)\right\},
\end{aligned}
$$

$$
\begin{array}{r}
d\left(x_{(m-1)(p+q)+q+r}, x_{n(p+q)+s}\right) \\
\leqslant h \max \left\{d\left(x_{(n-1)(p+q)+q+s}, x_{(m-1)(p+q)+r}\right),\right. \\
d\left(x_{(n-1)(p+q)+q+s}, x_{n(p+q)+s}\right), \\
d\left(x_{(m-1)(p+q)+r}, x_{(m-1)(p+q)+q+r}\right), \\
d\left(x_{(n-1)(p+q)+q+s}, x_{(m-1)(p+q)+q+r}\right), \\
\left.\quad d\left(x_{(m-1)(p+q)+r}, x_{n(p+q)+s}\right)\right\},
\end{array}
$$

and 


$$
\begin{aligned}
& d\left(x_{(n-1)(p+q)+p+s}, x_{m(p+q)+r}\right) \\
&<h \max \left\{d\left(x_{(n-1)(p+q)+s}, x_{(m-1)(p+q)+p+r}\right),\right. \\
& d\left(x_{(n-1)(p+q)+s}, x_{(n-1)(p+q)+p+s}\right), \\
& d\left(x_{(m-1)(p+q)+p+r}, x_{m(p+q)+r}\right), \\
& d\left(x_{(n-1)(p+q)+s}, x_{m(p+q)+r}\right), \\
&\left.d\left(x_{(m-1)(p+q)+p+r}, x_{(n-1)(p+q)+p+s}\right)\right\} .
\end{aligned}
$$

An examination of the right-hand sides of inequalities (254)-(258) shows that the smallest and largest subscripts present are $(n-1)(p+q)$ $+s$ and $m(p+q)+r$, respectively. Therefore (253) can be written

$$
d\left(x_{j}, x_{k}\right)<h^{2} \delta\left(O\left(x_{(n-1)(p+q)+s},(m-n+1)(p+q)+r-s\right)\right) .
$$

There exist integers $a$ and $b$ satisfying $(n-1)(p+q)+s<a<b<$ $m(p+q)+r$ such that

$$
\begin{aligned}
\delta\left(O\left(x_{(n-1)(p+q)+s},(m-n+1)(p+q)+r-s\right)\right) & =d\left(x_{a}, x_{b}\right) \\
& =d\left(x_{t(p+q)+u}, x_{v(p+q)+w}\right),
\end{aligned}
$$

where $a=t(p+q)+u, b=v(p+q)+w$.

Repeating the argument which developed inequalities (253)-(258) we obtain

$$
d\left(x_{t(p+q)+u}, x_{v(p+q)+w}\right) \leqslant h^{2} \delta\left(O\left(x_{(t-1)(p+q)+u},(v-t+1)(p+q)+w-u\right) .\right.
$$

But $(t-1)(p+q)+u \geqslant(n-2)(p+q)+s$ and $(v-t+1)(p+q)+w$ $-u<(m-n+2)(p+q)+r-s$, so that

$$
\begin{aligned}
& \delta\left(O\left(x_{(t-1)(p+q)+u},(v-t+1)(p+q)+w-u\right)\right) \\
& \quad \leqslant \delta\left(O\left(x_{(n-2)(p+q)+s},(m-n+2)(p+q)+r-s\right)\right) .
\end{aligned}
$$

Therefore

$$
d\left(x_{j}, x_{k}\right)<h^{2 n} \delta\left(O\left(x_{s}, m(p+q)+r-s\right)\right) \leqslant h^{2 n} \delta\left(O\left(x_{0},(m+1)(p+q)\right) .\right.
$$

Consider $\delta\left(O\left(x_{0}, n\right)\right)$ for any integer $n$. There exist integers $c$ and $d$ such that $\delta\left(O\left(x_{0}, n\right)\right)=d\left(x_{c}, x_{d}\right)$, where $0 \leqslant c<d \leqslant n$. If $c \geqslant \max (p, q)$ an argument similar to that of (253) shows that $d\left(x_{c}, x_{d}\right)<h \delta\left(O\left(x_{e}, m\right)\right)$ where $e$ satisfies $0<e<\max (p, q)$ and $m-e<d-c$. Consequently $\delta\left(O\left(x_{0}, n\right)\right)$ $=d\left(x_{c}, x_{d}\right)$, where $c$ now satisfies $0<c<\max (p, q)$.

Suppose $d>\max (p, q)$. If $q \leqslant p$, then 


$$
\begin{gathered}
d\left(x_{c}, x_{d}\right) \leqslant d\left(x_{c}, x_{p}\right)+d\left(x_{p}, x_{d}\right) . \\
d\left(x_{p}, x_{d}\right)=d\left(f^{p}\left(x_{0}\right), f^{q}\left(x_{d-q}\right)\right) \\
\leqslant h \max \left\{d\left(x_{0}, x_{d-q}\right), d\left(x_{0}, x_{p}\right), d\left(x_{d-q}, x_{d}\right), d\left(x_{0}, x_{d}\right), d\left(x_{d-q}, x_{p}\right)\right\} \\
\leqslant h \delta\left(O\left(x_{0}, d\right)\right) \leqslant h \delta\left(O\left(x_{0}, n\right)\right)=h d\left(x_{c}, x_{d}\right) .
\end{gathered}
$$

Therefore $d\left(x_{c}, x_{d}\right) \leqslant d\left(x_{c}, x_{p}\right) /(1-h)$, and for any integer $n, \delta\left(O\left(x_{0}, n\right)\right)$ $<d\left(x_{c}, x_{p}\right) /(1-h)$.

If $q>p$, then we obtain $\delta\left(O\left(x_{0}, n\right)\right) \leqslant d\left(x_{c}, x_{q}\right) /(1-h)$. In either case, $\delta\left(O\left(x_{0}, n\right)\right) \leqslant d\left(x_{c}, x_{r}\right) /(1-h)$, where $r=\max (p, q)$.

Therefore $\left\{x_{n}\right\}$ is Cauchy, hence convergent. Call the limit $z$. We shall first show that $z$ is a fixed point of $f^{p}$.

$$
\begin{gathered}
d\left(f^{p}(z), z\right) \leqslant d\left(f^{p}(z), f^{q}\left(x_{n(p+q)}\right)\right)+d\left(x_{n(p+q)+q}, z\right) . \\
d\left(f^{p}(z), f^{q}\left(x_{n(p+q)}\right) \leqslant h \max \left\{d\left(z, x_{n(p+q)}\right), d\left(z, f^{p}(z)\right), d\left(x_{n(p+q)}, x_{n(p+q)+q}\right),\right.\right. \\
\left.d\left(z, x_{n(p+q)+q}\right), d\left(x_{n(p+q)}, f^{p}(z)\right)\right\} .
\end{gathered}
$$

Taking the limit as $n \rightarrow \infty$ in both inequalities we obtain $d\left(f^{p}(z), z\right)$ $<h d\left(z, f^{P}(z)\right)$, a contradiction unless $f^{P}(z)=z$. Similarly we can show that $z$ is also a fixed point of $f^{q}$.

Let $z$ and $w$ be two fixed points of $f^{p}$. Then $d(z, w)=d\left(f^{p}(z), f^{q}(w)\right)$ $\leqslant h d(z, w)$, so that $z=w$ and the fixed point of $f^{p}$ is unique. But $f^{p}(f(z))$ $=f\left(f^{p}(z)\right)=f(z)$ so that $z=f(z)$. Uniqueness follows from the lemma.

For (76)-(100), (76) appears in [14]. (78) is a special case of (103), which appears in [1]. We can establish the following.

THEOREM 12. Let $f \in(91)$ or (94), let $B \subset X$ with $f(B) \subset B$. If there exists $a z \in B$ and a positive integer $n=n(z)$ such that $f^{n}(z)=z$, then $z$ is the unique fixed point of $f$ in $B$, and $f^{n}\left(x_{0}\right) \rightarrow z$ for each $x_{0} \in B$.

Proof. Uniqueness follows from the lemma.

Let $r\left(x_{0}\right)=\max \left\{d\left(z, f^{p}\left(x_{0}\right) \mid 1 \leqslant p<n\right\}\right.$. For all $m>n, m=q n+s$, where $q \geqslant 1,0 \leqslant s<n$. Let $x_{0} \in B$, and assume $f^{m}\left(x_{0}\right) \neq z$ for each $m$. Let $f \in(91)$. Then

$$
\begin{aligned}
& d\left(f^{m}\left(x_{0}\right), z\right) \leqslant h \max \left\{d\left(f^{(q-1) n+s}\left(x_{0}\right), z\right),\right. \\
& \left.d\left(f^{(q-1) n+s}\left(x_{0}\right), f^{n}(z)\right), d\left(z, f^{m}\left(x_{0}\right)\right)\right\} .
\end{aligned}
$$

Since $f^{m}\left(x_{0}\right) \neq z$ for any $m$,

$$
d\left(f^{m}\left(x_{0}\right), z\right) \leqslant h d\left(f^{(q-1) n+s}\left(x_{0}\right), z\right) \leqslant h^{q} r\left(x_{0}\right) \rightarrow 0 \text { as } q \rightarrow \infty .
$$

If $f \in(94)$, then 


$$
\begin{aligned}
d\left(f^{m}\left(x_{0}, z\right)=\right. & d\left(f^{q n+s}\left(x_{0}\right), f^{n}(z)\right) \\
\leqslant & h \max \left\{d\left(f^{(q-1) n+s}\left(x_{0}\right), z\right),\right. \\
& {\left[d\left(f^{(q-1) n+s}\left(x_{0}\right), f^{m}\left(x_{0}\right)\right)+0\right] / 2, } \\
& {\left.\left[d\left(f^{(q-1) n+s}\left(x_{0}\right), f^{n}(z)\right)+d\left(z, f^{m}\left(x_{0}\right)\right)\right] / 2\right\} } \\
= & h M\left(x_{0}, m, z\right), \text { say . }
\end{aligned}
$$

Note that

$$
d\left(f^{(q-1) n+s}\left(x_{0}\right), f^{m}\left(x_{0}\right)\right) / 2 \leqslant\left[d\left(f^{(q-1) n+s}\left(x_{0}\right), z\right)+d\left(z, f^{m}\left(x_{0}\right)\right)\right] / 2 .
$$

If

$$
M\left(x_{0}, m, z\right)=\left[d\left(f^{(q-1) n+s}\left(x_{0}\right), z\right)+d\left(z, f^{m}\left(x_{0}\right)\right)\right] / 2
$$

then

$$
d\left(f^{m}\left(x_{0}\right), z\right)<d\left(f^{(q-1) n+s}\left(x_{0}\right), z\right) \leqslant M\left(x_{0}, m, z\right),
$$

which leads to the contradiction $d\left(f^{m}\left(z_{0}\right), z\right)<d\left(f^{m}\left(x_{0}\right), z\right)$. Therefore $M\left(x_{0}, m, z\right)=d\left(f^{(q-1) n+s}\left(x_{0}\right), z\right)$, so that $d\left(f^{m}\left(x_{0}\right), z\right)<h^{q} r\left(x_{0}\right) \rightarrow 0$ as $q$ $\rightarrow \infty$.

The special case of this lemma for $f \in(76)$ appears in [14].

For definitions (101) $-(125)$ the best result is (103), which appears in [1].

4. Locally contractive mappings. A mapping is called locally contractive if, in each of the definitions, one adds the restriction that $x$ and $y$ be close to each other. For example, for (3) (see [9]) the definition would read: there exists an $\varepsilon>0$ such that $0<d(x, y)<\varepsilon$ implies $d(f(x), f(y))<d(x, y)$.

THEOREM 13. Except possibly for (11), (36), (61), (86), and (111), the concept of locally contractive is not extendable beyond definition (3) and its counterparts (28), (53), (78), and (103).

Proof. Reich [25, p. 8] has shown that (4) is not extendable to locally contractive mappings.

Define $f: R \rightarrow R$ by $f(x)=x+\varepsilon$. Then $d(f(x), f(y))=d(x, y)<\varepsilon$ $=d(x, f(x))=d(y, f(y))$, and $f \in(6)$, but has no fixed points.

Now let $f(x)=x+\lambda, x \in R, \lambda=2 \varepsilon(1-h) / h$, for some $h$ satisfying $\frac{\xi}{3}<h<1$. Then $d(f(x), f(y))=d(x, y)$. We wish to show

$$
d(x, y)<h \max \{d(x, f(y)), d(y, f(x))\}=h(d(x, y)+\lambda)
$$


i.e., $(1-h) d(x, y)<h \lambda$. Since $d(x, y)<\varepsilon$, it will be sufficient to show that $(1-h) \varepsilon<h \lambda$. This last inequality holds for the above choice of $\lambda$. Thus $f \in(12) . f$ also satisfies (13), (14), and (18), but $f$ has no fixed points. An examination of Theorem 1 shows that the remaining definitions have been taken care of.

5. Pairs of mappings. A number of definitions exist, in the literature, which define a contractive type condition between a pair of functions $f, g: X \rightarrow X$. Applying this principle yields definitions (126)-(250). For example, (129) (see [17]) takes the form

(129) There exists a number $a, 0<a<\frac{1}{2}$, such that for each $x, y \in X$,

$$
d(f(x), g(y)) \leqslant a[d(x, f(x))+d(y, g(y))] .
$$

(126) appears in [23], (136) in [5], (140) in [26], (143') in [29], (148) in [34], (182) in [35], and (182) with $c=0$ in [12].

We now list the most general fixed point theorems for each of the classes (126)-(150), etc.

THEOREM 14. Let $f, g \in$ (146), $x_{0} \in X$. Then $f$ and $g$ have a common unique fixed point $z$ and $(f g)^{n}\left(x_{0}\right) \rightarrow z$ and $(g f)^{n}\left(x_{0}\right) \rightarrow z$.

Proof. Let $x_{0} \in X$. Define the sequence $\left\{x_{n}\right\}$ by $x_{2 n+1}=f\left(x_{2 n}\right), x_{2 n+2}$ $=g\left(x_{2 n+1}\right)$. Assume $x_{n} \neq x_{n+1}$ for each $n$.

$$
\begin{aligned}
d\left(x_{2 n+1}, x_{2 n+2}\right)= & d\left(f\left(x_{2 n}\right), g\left(x_{2 n+1}\right)\right) \\
\leqslant & h \max \left\{d\left(x_{2 n}, x_{2 n+1}\right), d\left(x_{2 n}, x_{2 n+1}\right),\right. \\
& \left.\quad d\left(x_{2 n+1}, x_{2 n+2}\right), \frac{1}{2}\left[d\left(x_{2 n}, x_{2 n+2}\right)+0\right]\right\} \\
= & h M\left(x_{2 n}, x_{2 n+1}\right) .
\end{aligned}
$$

$M\left(x_{2 n}, x_{2 n+1}\right)=d\left(x_{2 n}, x_{2 n+2}\right) / 2$ leads to the contradiction $d\left(x_{2 n+1}, x_{2 n+2}\right)$ $\leqslant h d\left(x_{2 n+1}, x_{2 n+2}\right)$. Therefore $d\left(x_{2 n+1}, x_{2 n+2}\right) \leqslant h d\left(x_{2 n}, x_{2 n+1}\right)$. Similarly, $d\left(x_{2 n}, x_{2 n+1}\right) \leqslant h d\left(x_{2 n-1}, x_{2 n}\right)$, so that $d\left(x_{2 n+1}, x_{2 n+2}\right) \leqslant h^{2} d\left(x_{2 n-1}, x_{2 n}\right)$ $\leqslant \cdots \leqslant h^{2 n} d\left(x_{1}, x_{2}\right)$ and $d\left(x_{2 n}, x_{2 n+1}\right) \leqslant h^{2 n} d\left(x_{0}, x_{1}\right)$.

Let $r\left(x_{0}\right)=\max \left\{d\left(x_{0}, x_{1}\right), d\left(x_{1}, x_{2}\right)\right\}$. For any $m>n$,

$$
\begin{aligned}
d\left(x_{m}, x_{n}\right) & =\sum_{k=0}^{m-n-1} d\left(x_{n+k}, x_{n+k+1}\right) \\
& \leqslant \sum_{k=0}^{m-n-1} h^{2(n+k)} r\left(x_{0}\right) \leqslant h^{2 n} r\left(x_{0}\right)\left(1-h^{2}\right)^{-1} .
\end{aligned}
$$

$\left\{x_{n}\right\}$ is Cauchy, hence convergent. Call the limit $z$, 


$$
\begin{gathered}
d(f(z), z) \leqslant d\left(f(z), x_{2 n+2}\right)+d\left(x_{2 n+2}, z\right) . \\
d\left(f(z), x_{2 n+2}\right) \leqslant h \max \left\{d\left(z, x_{2 n+1}\right), d(z, f(z)), d\left(x_{2 n+1}, x_{2 n+2}\right),\right. \\
\frac{1}{2}\left[d\left(z, x_{2 n+2}\right)+d\left(x_{2 n+1}, f(z)\right]\right\} .
\end{gathered}
$$

Taking the limit as $n \rightarrow \infty$ we obtain $d(f(z), z)<h d(f(z), z)$, which implies $f(z)=2$. Similarly, $g(z)=z$. Suppose $z$ and $w$ are two fixed points of $f$ and $g$. Then

$$
d(z, w)=d(f(z), g(w)) \leqslant h \max \left\{d(z, w), 0,0, \frac{1}{2}[d(z, w)+d(w, z)]\right\},
$$

and $d(z, w) \leqslant h d(z, w)$, which implies $z=w$.

Define $\left\{y_{n}\right\}$ by $y_{0}=x_{0}, y_{2 n+1}=g\left(x_{2 n}\right), y_{2 n+2}=f\left(x_{2 n+1}\right)$. Then it can be shown that $\left\{y_{n}\right\}$ is Cauchy, hence convergent. $y_{n} \rightarrow z$ by the uniqueness of the fixed point.

THEOREM 15. Let $f, g$ be continuous and satisfy (147). If either $\left\{(f g)^{n}\left(x_{0}\right)\right\}$ or $\left\{(g f)^{n}\left(x_{0}\right)\right\}$ has a cluster point $z$, then $z$ is the unique fixed point of $f$ and $g$, and $(f g)^{n}\left(x_{0}\right) \rightarrow z$ or $(g f)^{n}\left(x_{0}\right) \rightarrow z$.

PROOF. For each $x \neq f(x)$,

$$
\begin{array}{r}
d(f(x), g(f(x)))<\max \{d(x, f(x)), d(x, f(x)), d(f(x), g(f(x))), \\
[d(x, g(f(x)))+d(f(x), f(x))] / 2\} .
\end{array}
$$

If the maximum of the right-hand side is $d(x, g(f(x))) / 2$, we are led to the contradiction $d(f(x), g(f(x)))<d(f(x), g(f(x)))$. Therefore $d(f(x), g(f(x)))$ $<d(x, f(x))$. Similarly, $x \neq g(x)$ implies $d(f(g(x)), g(x))<d(x, g(x))$. Thus

$$
d(f(g(f(x))), g(f(x)))<d(f(x), g(f(x)))<d(x, f(x)) .
$$

Let $z$ be a cluster point of $\left\{(g f)^{n}\left(x_{0}\right)\right\}$. We shall assume that $(g f)^{n}\left(x_{0}\right)$ $\neq(g f)^{n+1}\left(x_{0}\right)$ for each $n \geqslant 0$ and that $z \neq f(z), z \neq g(z)$, and $z \neq g(f(z))$. If we define $V(x)=d(x, f(x))$, then from (259), $V(g(f(x))) \leqslant V(x)$ and, for $x \neq z$ we have strict inequality. By Lemma 1 of $[31], g(f(z))=z$.

Suppose $f(z) \neq z$. Then $d(f(z), z)=d(f(z), g(f(z)))<d(z, f(z))$, a contradiction. Similarly, $g(z)=z$. Uniqueness of $z$ is easy.

Assume $(g f)^{n}\left(x_{0}\right) \neq z$ for any $n$. Then

$$
\begin{aligned}
d\left((g f)^{n}\left(x_{0}\right), z\right)= & d\left(g\left(f(g f)^{n-1}\left(x_{0}\right)\right), f(z)\right) \\
<\max \left\{d\left(z, f\left((g f)^{n-1}\left(x_{0}\right)\right)\right), d(z, f(z)),\right. & \\
& \quad d\left(f\left((g f)^{n-1}\left(x_{0}\right)\right),(g f)^{n}\left(x_{0}\right)\right), \\
& {\left[d\left(z,(g f)^{n}\left(x_{0}\right)\right)+d\left(f\left((g f)^{n-1}\left(x_{0}\right)\right), z\right] / 2\right\}, }
\end{aligned}
$$


or

$$
\begin{aligned}
d\left((g f)^{n}\left(x_{0}\right), z\right)<\max \left\{d\left(z, f\left((g f)^{n-i}\left(x_{0}\right)\right)\right),\right. \\
d\left(f\left((g f)^{n-1}\left(x_{0}\right),(g f)^{n}\left(x_{0}\right)\right)\right\}
\end{aligned}
$$

$$
\begin{aligned}
d\left(z, f\left((g f)^{n-1}\left(x_{0}\right)\right)\right)<\max \{ & d\left((g f)^{n-1}\left(x_{0}\right), z\right) \\
& d\left((g f)^{n-1}\left(x_{0}\right), f\left((g f)^{n-1}\left(x_{0}\right)\right)\right), 0 \\
& {\left.\left[d\left((g f)^{n-1}\left(x_{0}\right), z\right)+d\left(z, f\left((g f)^{n-1}\left(x_{0}\right)\right)\right)\right] / 2\right\} }
\end{aligned}
$$

which leads to

$$
\begin{aligned}
d\left(z, f\left((g f)^{n-1}\left(x_{0}\right)\right)\right) & \\
& <\max \left\{d\left(z,(g f)^{n-1}\left(x_{0}\right)\right), d\left((g f)^{n-1}\left(x_{0}\right), f\left((g f)^{n-1}\left(x_{0}\right)\right)\right)\right\}
\end{aligned}
$$

Since

$$
d\left(f\left((g f)^{n-1}\left(x_{0}\right)\right),(g f)^{n}\left(x_{0}\right)\right)<d\left((g f)^{n-1}\left(x_{0}\right) f\left((g f)^{n-1}\left(x_{0}\right)\right)\right),
$$

we have

$$
d\left(z,(g f)^{n}\left(x_{0}\right)\right)<\max \left\{d\left(z,(g f)^{n-1}\left(x_{0}\right)\right), d\left((g f)^{n-1}\left(x_{0}\right), f\left((g f)^{n-1}\left(x_{0}\right)\right)\right)\right\} .
$$

Since $(g f)^{n_{i}}\left(x_{0}\right) \rightarrow z$, and $f$ and $g$ are continuous,

$$
d\left((g f)^{n_{i}}\left(x_{0}\right), f\left((g f)^{n_{i}}\left(x_{0}\right)\right)\right) \rightarrow d(z, f(z))=0 .
$$

Fix $\varepsilon>0$, and pick $n_{j}$ so that $d\left(z,(g f)^{n_{j}}\left(x_{0}\right)\right)<\varepsilon$ and $d\left((g f)^{n_{j}}\left(x_{0}\right)\right.$, $\left.f\left((g f)^{n_{j}}\left(x_{0}\right)\right)\right)<\varepsilon$. Then, for $n>n_{j}$,

$$
\begin{aligned}
d\left(z,(g f)^{n}\left(x_{0}\right)\right) & <\max \left\{d\left(z,(g f)^{n-2}\left(x_{0}\right)\right), d\left((g f)^{n-2}\left(x_{0}\right), f\left((g f)^{n-2}\left(x_{0}\right)\right)\right)\right. \\
& \left.d\left((g f)^{n-1}\left(x_{0}\right), f\left((g f)^{n-1}\left(x_{0}\right)\right)\right)\right\} \\
& =\max \left\{d\left(z,(g f)^{n-2}\left(x_{0}\right)\right), d\left((g f)^{n-2}\left(x_{0}\right), f\left((g f)^{n-2}\left(x_{0}\right)\right)\right)\right\} \\
& <\cdots \\
& <\max \left\{d\left(z,(g f)^{n_{j}}\left(x_{0}\right)\right), d\left((g f)^{n_{j}}\left(x_{0}\right), f\left((g f)^{n_{j}}\left(x_{0}\right)\right)\right)\right\}<\varepsilon .
\end{aligned}
$$

A similar argument applies when $\left\{(f g)^{n}\left(x_{0}\right)\right\}$ has a cluster point. 
THEOREM 16. Let $f \in(148)$ with the $\alpha_{i}$ satisfying (i) of Theorem 10. Let $x_{0} \in X$. Then $(f g)^{n}\left(x_{0}\right)$ and $(g f)^{n}\left(x_{0}\right)$ converge. If, in addition, condition (ii) of Theorem 10 is satisfied, then $f$ and $g$ have a common unique fixed point $z$ and $(f g)^{n}\left(x_{0}\right) \rightarrow z,(g f)^{n}\left(x_{0}\right) \rightarrow z$.

Proof. Define the sequence $\left\{x_{n}\right\}$ by $x_{0} \in X, x_{2 n+1}=f\left(x_{2 n}\right), x_{2 n+2}$ $=g\left(x_{2 n+1}\right), n \geqslant 0$. Let $b_{n}=d\left(x_{n}, x_{n+1}\right)$ for each $n$, and assume $b_{n}>0$ for each $n$.

As in the proof of Theorem 10, we obtain $b_{2 n+1} \leqslant r\left(b_{2 n}\right) b_{2 n}$ and $b_{2 n+2}$ $\left\langle s\left(b_{2 n+1}\right) b_{2 n+1}\right.$. Thus $\left\{b_{2 n+1}\right\}$ and $\left\{b_{2 n}\right\}$ are monotone decreasing, hence convergent. Call the limits $c_{1}$ and $c_{2}$, respectively.

Assume $c_{1}>0$, and set $q=r\left(c_{1}\right) s\left(c_{1}\right)$. Then $b_{2 n+1}>c_{1}$ implies $r\left(b_{2 n+1}\right) s\left(b_{2 n+1}\right) \leqslant q$ for all $n$, and $b_{2 n+1} \leqslant q b_{2 n-1}<\cdots<q^{n} b_{1} \rightarrow 0$. Similarly, $c_{2}=0$.

The same argument as that of Theorem 4 shows that $\left\{x_{n}\right\}$ is Cauchy, hence convergent. Call the limit $z$. Similarly $\left\{y_{n}\right\}$ converges, where $y_{0}=x_{0}, y_{2 n+1}$ $=g\left(y_{2 n}\right), y_{2 n+2}=f\left(y_{2 n+1}\right), n \geqslant 0$.

If (ii) is satisfied, then, as in (251), assuming $x_{n} \neq z$ for any $n$, $d\left(f(z), x_{2 n+2}\right)$

$$
\leqslant \frac{\left(\alpha_{1}+\alpha_{3}\right) d\left(z, x_{2 n+2}\right)+\left(\alpha_{2}+\alpha_{4}\right) d\left(x_{2 n+1}, x_{2 n+2}\right)+\alpha_{5} d\left(z, x_{2 n+1}\right)}{1-\alpha_{1}-\alpha_{4}} .
$$

Taking the limit as $n \rightarrow \infty$ yields $z=f(z)$. Similarly, $d\left(x_{2 n+1}, g(z)\right) \rightarrow 0$ as $n \rightarrow \infty$, so that $z=g(z)$.

Suppose $z=f(z), w=g(w), z \neq w$. Then, from (148), $d(z, w)<\left(\alpha_{3}+\alpha_{4}\right.$ $\left.+\alpha_{5}\right) d(z, w)<d(z, w)$, a contradiction.

Therefore $f$ and $g$ have the same fixed points. Suppose $f$ has two distinct fixed points $z$ and $w$. Then $d(z, w)=d(f(z), g(w)) \leqslant\left(\alpha_{3}+\alpha_{4}+\alpha_{5}\right) d(z, w)$ $<d(z, w)$, a contradiction.

A similar argument applies to $\left\{y_{n}\right\}$.

Theorem 16 is an improvement over [34, Theorem 2].

THEOREM 17. Let $f, g \in(171)$. Then $f$ and $g$ have a common fixed point $z$ and $\left(f^{p} g^{q}\right)^{n}\left(x_{0}\right) \rightarrow z,\left(g^{q} f^{p}\right)^{n}\left(x_{0}\right) \rightarrow z$ for each $x_{0} \in X$.

THEOREM 18. Let $f, g \in(172), f, g$ continuous. If $z$ is a cluster point of $\left\{\left(f^{p} g^{q}\right)^{n}\left(x_{0}\right)\right\}$ or $\left\{\left(g^{q} f^{p}\right)^{n}\left(x_{0}\right)\right\}$ for some $x_{0} \in X$, then $z$ is the unique fixed point of $f$ and $g$, and $\left(f^{p} g^{q}\right)^{n}\left(x_{0}\right) \rightarrow z$ or $\left(g^{q} f^{p}\right)^{n}\left(x_{0}\right) \rightarrow z$.

THEOREM 19. Let $f, g \in(173)$, with the $\alpha_{i}$ satisfying Theorem 10. Then $\left(f^{p} g^{q}\right)^{n}\left(x_{0}\right)$ and $\left(g^{q} f^{p}\right)^{n}\left(x_{0}\right)$ converge. If, in addition, condition (ii) of Theorem 10 is satisfied, then $f$ and $g$ have a common unique fixed point $z$ and $\left(f^{p} g^{q}\right)^{n}\left(x_{0}\right)$ $\rightarrow z,\left(g^{q} f^{p}\right)^{n}\left(x_{0}\right) \rightarrow z$. 
These three theorems are proved by replacing $f$ by $f^{p}, g$ by $g^{q}$ in Theorems $14-16$.

6. Sequences of mappings. There are three types of theorems for sequences of mappings. The first assumes that each pair $f_{i}, f_{j}$ satisfies the same contractive condition, and concludes that $\left\{f_{n}\right\}$ has a common fixed point. The second assumes that each $f_{n}$ satisfies the same contractive condition and that $\left\{f_{n}\right\}$ tends pointwise to a limit function $f$. The conclusion is that $f$ has a fixed point $z$ which is the limit of each of the fixed points $z_{n}$ of $f_{n}$. The third type assumes that each $f_{n}$ has a fixed point $z_{n}$, and that $\left\{f_{n}\right\}$ converges uniformly to a function $f$ which satisfies a particular contractive condition. With $z$ the fixed point of $f$, the conclusion is that $z_{n} \rightarrow z$.

THEOREM 20. Let $0 \leqslant h<1$. Let $\left\{f_{n}\right\}$ be a sequence of functions satisfying

$$
\begin{aligned}
d\left(f_{i}^{p}(x), f_{j}^{p}(y)\right) \leqslant h \max \{d(x, y), & d\left(x, f_{i}^{p}(x)\right), d\left(y, f_{j}^{p}(y)\right), \\
& {\left.\left[d\left(x, f_{j}^{p}(y)\right)+d\left(y, f_{i}^{p}(x)\right)\right] / 2\right\} }
\end{aligned}
$$

for each $x, y \in X$, and some fixed integer $p$. Then $\left\{f_{n}\right\}$ has a unique common fixed point $z$.

Proof. Set $S=f_{i}^{p}, T=f_{j}^{p}$. Then $S$ and $T$ satisfy Theorem 14, so that $S$ and $T$ have a unique common fixed point $z$. Since each pair of functions $f_{i}^{p}, f_{j}^{p}$ has a unique common fixed point, the sequence $\left\{f_{n}^{p}\right\}$ has this property. But $z$ a fixed point of $f_{n}^{p}$ implies $z$ is a fixed point of $f_{n}$, so that $z$ is the unique common fixed point.

Theorem 1 of [6] is a special case of Theorem 20. Theorem 20 shows that the hypotheses of continuity and pairwise commuting of $f_{i}$ and $f_{j}$ can be omitted from Theorem 1 of [6]. Theorem 1 of [22] and Theorem 1 of [16] are also special cases of Theorem 20.

THEOREM 21. Let $\alpha_{i}(t)$ be decreasing functions, $\alpha_{i}:(0, \infty) \rightarrow[0,1)$ satisfying $\sum_{i=1}^{5} \alpha_{i}(t)<1$ for each $t>0$, and condition (i) and (ii) of Theorem 10. Let $\left\{f_{n}\right\}$ be a sequence of functions satisfying

$$
\begin{aligned}
d\left(f_{i}^{p}(x), f_{j}^{p}(y)\right) \leqslant & \alpha_{1} d\left(x, f_{i}^{p}(x)\right)+\alpha_{2} d\left(y, f_{j}^{p}(y)\right)+\alpha_{3} d\left(x, f_{j}^{p}(y)\right) \\
& +\alpha_{4} d\left(y, f_{i}^{p}(x)\right)+\alpha_{5} d(x, y)
\end{aligned}
$$

for each pair $x, y \in X, x \neq y$, where $\alpha_{i}=\alpha_{i}(d(x, y))$. Then $\left\{f_{n}\right\}$ has a unique common fixed point $z$.

Set $S=f_{i}^{p}, T=f_{j}^{p}$. Then $S$ and $T$ satisfy Theorem 16 . The rest of the argument is the same as that for Theorem 20.

Theorem 4 of [25] is a special case of Theorem 21 . 
THEOREM 22. Let $\left\{f_{n}\right\}$ be a sequence of functions satisfying (23),.. each $n$, for the same functions $\alpha_{i}(t)$, and such that $\left\{f_{n}\right\}$ tends pointwise to a function $f$. Ther $f$ has $a$ unique fixed point $z$ and $z_{n} \rightarrow z$, where $z_{n}$ are the fixed points of $f_{n}$.

Proof. Since $f \in(23)$, for each pair $x, y \in X, x \neq y$,

$$
\begin{aligned}
d\left(f_{n}(x), f_{n}(y)\right) \leqslant & \alpha_{1} d\left(x, f_{n}(x)\right)+\alpha_{2} d\left(y, f_{n}(y)\right)+\alpha_{3} d\left(x, f_{n}(y)\right) \\
& +\alpha_{4} d\left(y, f_{n}(x)\right)+\alpha_{5} d(x, y),
\end{aligned}
$$

where $\alpha_{i}=\alpha_{i}(d(x, y))$. Taking the limit as $n \rightarrow \infty$ shows that $f \in$ (23). From Theorem $4, f$ has a unique fixed point $z$. It remains to show that $z_{n} \rightarrow z$.

$z_{n} \neq z$ implies $d\left(z_{n}, z\right)=d\left(f_{n}\left(z_{n}\right), f(z)\right) \leqslant\left(\alpha_{3}+\alpha_{4}+\alpha_{5}\right) d\left(z_{n}, z\right)$ $<d\left(z_{n}, z\right)$, a contradiction.

Theorem 3 of [15] and Theorem 6 of [24] are special cases of Theorem 22.

THEOREM 23. Let $\left\{f_{n}\right\}$ be a sequence of functions satisfying (24) for each $n$ and the same $h$, such that $\left\{f_{n}\right\}$ tends pointwise to a function $f$. Then $f$ has a unique fixed point $z$, and $z_{n} \rightarrow z$, where the $z_{n}$ are fixed points of $f_{n}$.

Proof. For each $x, y \in X$, and each $n$,

$$
\begin{array}{r}
d\left(f_{n}(x), f_{n}(y)\right) \leqslant h \max \left\{d(x, y), d\left(x, f_{n}(x)\right), d\left(y, f_{n}\left(y^{\prime}\right)\right),\right. \\
\left.d\left(x, f_{n}(y)\right), d\left(y, f_{n}(x)\right)\right\} .
\end{array}
$$

Taking the limit as $n \rightarrow \infty$, and using the fact that $d$ is continuous it follows that $f \in(24)$, and hence has a unique fixed point. Call it $z$.

$$
d\left(z_{n}, z\right)=d\left(f_{n}\left(z_{n}\right), f(z)\right) \leqslant d\left(f_{n}\left(z_{n}\right), f_{n}(z)\right)+d\left(f_{n}(z), f(z)\right) .
$$

But $d\left(f_{n}\left(z_{n}\right), f_{n}(z)\right) \leqslant h \max \left\{d\left(z_{n}, z\right), d\left(z, f_{n}(z)\right)\right\}$. Therefore

$$
d\left(z_{n}, z\right) \leqslant \max \left\{(1-h)^{-1}, 1+h\right\} d\left(z, f_{n}(z)\right) \rightarrow 0 \text { as } n \rightarrow \infty .
$$

THEOREM 24 ([15, THEOREM 4]). Let $\left\{f_{n}\right\}$ be a sequence of self-mappings of $X$ with fixed points $z_{n}, n=1,2, \ldots$, and such that $f_{n} \rightarrow f$ uniformly, where $f: X \rightarrow X$ and satisfies (23) with fixed point 2 . Then $z_{n} \rightarrow z$.

[24, Theorem 4] is a special case of Theorem 24.

THEOREM 25. Let $\left\{f_{n}\right\}$ be a sequence of self-mappings of $X$ with fixed points $z_{n}, n=1,2, \ldots$, such that $f_{n} \rightarrow f$ uniformly, where $f: X \rightarrow X$ and $f$ satisfies (24) with fixed point $z$. Then $z_{n} \rightarrow z$.

Proof. Fix $\varepsilon>0$. From the uniform convergence of $\left\{f_{n}\right\}$, there exists an integer $N$ such that, for all $n \geqslant N, d\left(f_{n}(x), f(x)\right)<\varepsilon / M$ for all $x \in X$, where 
$M=\max \left\{1+h,(1-h)^{-1}\right\} . \quad d\left(z_{n}, z\right)=d\left(f_{n}\left(z_{n}\right), f(z)\right) \leqslant d\left(f_{n}\left(z_{n}\right), f\left(z_{n}\right)\right)$ $+d\left(f\left(z_{n}\right), f(z)\right)$. Since $f \in(24)$,

$$
d\left(f\left(z_{n}\right), f(z)\right) \leqslant h \max \left\{d\left(z_{n}, f\left(z_{n}\right)\right), d\left(z_{n}, z\right)\right\} .
$$

For each $n$ such that the maximum equals $d\left(z_{n}, f\left(z_{n}\right)\right)$, it follows that $d\left(z_{n}, z\right) \leqslant(1+h) d\left(z_{n}, f\left(z_{n}\right)\right)$. For each $n$ such that the maximum equals $d\left(z_{n}, z\right)$, it follows that $d\left(z_{n}, z\right) \leqslant d\left(z_{n}, f\left(z_{n}\right)\right) /(1-h)$. In either case, $n>N$ implies $d\left(z_{n}, z\right)<\varepsilon$.

[5, Theorem 7], [16, Theorem 2], [20, Theorem 2], and [32, Theorem 3] are special cases of both Theorems 24 and 25.

7. Some extensions. In certain instances it is possible to extend some of the contractive definitions to allow equality. For example, Kannan [17] extended (4) to allow $a=\frac{1}{2}$. With the added hypotheses that $f$ be continuous and $X$ be compact, he showed that $f$ has a fixed point. Reich [27] and Soardi [28] showed that the assumption of continuity is superfluous. The most general theorem of this type is the following, which includes nonexpansive mappings as a special case.

THEOREM 27. Let $X$ be a uniformly convex Banach space, $K$ a nonempty, closed, convex subset of $X$ such that $f: K \rightarrow K$ and $f \in(21)$ with $\lambda=1$. If there exists a point $x \in K$ with bounded orbit, and if $\sup _{x, y \in K^{s}}(x, y)<1$, then $f$ has a fixed point.

Proof. Let $O(x)=\left\{x, f(x), \ldots, f^{n}(x), \ldots\right\}$.

We shall first show that

$$
\delta(O(x))=\sup _{n} d\left(x, f^{n}(x)\right)=g(x),
$$

say. For each $n>0$,

$$
\begin{aligned}
d\left(f^{n}(x), f^{n+1}(x)\right) \leqslant & q d\left(f^{n-1}(x), f^{n}(x)\right)+r d\left(f^{n-1}(x), f^{n}(x)\right) \\
& +s d\left(f^{n}(x), f^{n+1}(x)\right)+t\left[d\left(f^{n-1}(x), f^{n+1}(x)\right)+0\right],
\end{aligned}
$$

so that

$$
\begin{aligned}
d\left(f^{n}(x), f^{n+1}(x)\right) & \leqslant(q+r+t)(1-s-t)^{-1} d\left(f^{n-1}(x), f^{n}(x)\right) \\
& \leqslant d\left(f^{n-1}(x), f^{n}(x)\right),
\end{aligned}
$$

provided $\sup _{x, y \in K}(s+t)<1$.

If $\sup \{s(x, y)+t(x, y) \mid x, y \in X\}=1$, then one can find $x_{n}, y_{n} \in X$ for each $n \in N$ such that $s\left(x_{n}, y_{n}\right)+t\left(x_{n}, y_{n}\right) \rightarrow 1$ as $n \rightarrow \infty$. Thus $r\left(x_{n}, y_{n}\right)$ 
$+q\left(x_{n}, y_{n}\right)+t\left(x_{n}, y_{n}\right) \rightarrow 0$, so that $t\left(x_{n}, y_{n}\right) \rightarrow 0$. But then $s\left(x_{n}, y_{n}\right)-1 \rightarrow 0$, a contradiction.

By induction, one can show that $d\left(f(x), f^{n+1}(x)\right) \leqslant g(x)$, and then that $d\left(f^{n}(x), f^{m}(x)\right) \leqslant g(x)$ for each $n \geq 0$, each $m>n$.

Define $C_{0}(x)=O(x), C_{n}(x)=\overline{c o} f\left(C_{n-1}(x)\right), n>0$.

We wish to show that, for each $n \geqslant 0, \delta\left(C_{n}(x) \cup C_{n+1}(x)\right)<g(x)$. The proof will be by induction. The proof for $n=0$ differs little from that for general $n$, and will therefore be omitted. Assume the induction hypothesis.

Let $z \in C_{n}(x), y \in C_{n}(x) \cup C_{n+1}(x)$. Fix $\varepsilon>0$. If $y \in C_{n}(x)$, then there exist $y_{1}, y_{2}, \ldots, y_{N} \in C_{n-1}(x)$ and $\lambda_{1}, \ldots, \lambda_{N}, \lambda_{i} \geqslant 0, \sum_{i=1}^{N} \lambda_{i}=1$ such that $d\left(\sum_{i=1}^{N} \lambda_{i} f\left(y_{i}\right), y\right)<\varepsilon$.

$$
\begin{aligned}
d(y, f(z)) \leqslant & \varepsilon+d\left(\sum_{i=1}^{N} \lambda_{i} f\left(y_{i}\right), f(z)\right) \leqslant \varepsilon+d\left(\sum_{i=1}^{N} \lambda_{i} f\left(y_{i}\right), \sum_{i=1}^{N} \lambda_{i} f(z)\right) \\
\leqslant & \varepsilon+\max _{1<i<N} d\left(f\left(y_{i}\right), f(z)\right) \leqslant \varepsilon+d\left(f\left(y_{j}\right), f(z)\right) \\
\leqslant & \varepsilon+q d\left(y_{j}, z\right)+r d\left(y_{j}, f\left(y_{j}\right)\right)+s d(z, f(z)) \\
& +t\left[d\left(y_{j}, f(z)\right)+d\left(z, f\left(y_{j}\right)\right)\right],
\end{aligned}
$$

where $q, r, s, t$ are evaluated at $\left(y_{j}, z\right)$.

$$
\begin{aligned}
d(y, f(z)) \leqslant & \varepsilon+(q+r+t) g(x) \\
& +s \sup _{y \in C_{n}(x)} d(y, f(z))+t \sup _{y \in C_{n}(x)} d(y, f(z)) .
\end{aligned}
$$

The right-hand side of the above inequality is now independent of $y$ so that we have, since $\varepsilon$ is arbitrary,

$$
\sup _{y \in C_{n}(x)} d(y, f(z)) \leqslant(q+r+t)(1-s-t)^{-1} g(x) \leqslant g(x) .
$$

If $y \in C_{n+1}(x)$, then for each $\varepsilon>0$ there exist $y_{1}, \ldots, y_{N} \in C_{n}(x), \lambda_{i} \geqslant 0$, $\sum_{i=1}^{N} \lambda_{i}=1$ such that $d\left(\sum_{i=1}^{N} \lambda_{i} f\left(y_{i}\right), y\right)<\varepsilon$.

$$
\begin{aligned}
d(y, f(z)) \leqslant & \varepsilon+d\left(\sum_{i=1}^{N} \lambda_{i} f\left(y_{i}\right), f(z)\right) \\
\leqslant & \varepsilon+q d\left(y_{j}, z\right)+r d\left(y_{j}, f\left(y_{j}\right)\right)+s d(z, f(z)) \\
& +t\left[d\left(y_{j}, f(z)\right)+d\left(z, f\left(y_{j}\right)\right)\right] .
\end{aligned}
$$

$d\left(z, f\left(y_{j}\right)\right) \leqslant g(x)$ by what we have just proved. Thus

$$
d(y, f(z)) \leqslant \varepsilon+(q+r+t) g(x)+(s+t) \sup _{y \in C_{n+1}(x)} d(y, f(z)) .
$$

Since $\varepsilon$ is arbitrary, 


$$
\sup _{y \in C_{n+1}(x)} d(y, f(z)) \leqslant(q+r+t)(1-s-t)^{-1} g(x) \leqslant g(x) .
$$

Therefore

$$
\sup _{y \in C_{n}(x) \cup C_{n+1}(x)} d(y, f(z)) \leqslant g(x) .
$$

Now let $z \in \overline{\operatorname{co}} f\left(C_{n}(x)\right), y \in C_{n}(x) \cup C_{n+1}(x)$. For each $\varepsilon>0$ there exist $z_{1}, \ldots, z_{N} \in C_{n+1}(x)$ and scalars $\lambda_{i} \geqslant 0, \sum_{i=1}^{N} \lambda_{i}=1$ such that $d\left(\sum_{i=1}^{N} \lambda_{i} z_{i}, z\right)<\varepsilon$. Then

$$
d(y, z) \leqslant \varepsilon+d\left(\sum_{i=1}^{N} \lambda_{i} z_{i}, y\right) \leqslant \varepsilon+d\left(z_{j}, y\right) \leqslant \varepsilon+g(x) .
$$

Since $\varepsilon$ is arbitrary, the result is proved.

Let $x_{0} \in K$ be a point with bounded orbit. As in the proof of Theorem i vi [28], it is possible to construct a Cauchy sequence $\left\{x_{n}\right\}$. Call the limit $z$. It then follows from [28] that $f\left(x_{n}\right) \rightarrow z$,

$$
\begin{aligned}
d(z, f(z)) \leqslant & d\left(z, f\left(x_{n}\right)\right)+d\left(f\left(x_{n}\right), f(z)\right) \\
\leqslant & d\left(z, f\left(x_{n}\right)\right)+q d\left(x_{n}, z\right)+r d\left(x_{n}, f\left(x_{n}\right)\right)+s d(z, f(z)) \\
& +t\left[d\left(x_{n}, f(z)\right)+d\left(z, f\left(x_{n}\right)\right)\right] .
\end{aligned}
$$

Taking $\lim _{n}$ we get $d(z, f(z)) \leqslant(s+t) d(z, f(z))$, which implies $z=f(z)$.

Theorem 27, with $r=s$, appears in [19]. With $t=0, r=s, q, r, s$ constants, Theorem 27 reduces to Theorem 1 of [28]. For $q, r, s, t$ constants, $r=s$, we get a generalization of Theorem 2 of [11], demonstrating that the hypothesis in [11] of continuity of $f$ is superfluous. Also the boundedness of $K$ can be weakened to having a point in $K$ with bounded orbit.

A companion theorem is the following.

THEOREM 28. Let $X$ be a Banach space, $K$ weakly compact, $f$ a continuous selfmapping of $K$ satisfying (21) with $\lambda=1$, and

$$
\sup _{x, y \in K} q(x, y)<1, \quad \sup _{x, y \in K} s(x, y)<1 .
$$

Then $f$ has a fixed point.

The proof of Theorem 28 parallels that of Theorem 2 in [28].

THEOREM 29. Let $X$ be a strictly convex space, $f: K \rightarrow K, f \in\left(19^{\prime}\right)$ with $\lambda=1$, and $\inf _{x, y \in K} a(x, y)>0$. Then the set of fixed points of $f$ is closed and convex.

Proof. Let $U$ be the set of fixed points of $f$ in $K,\left\{x_{n}\right\}$ a Cauchy sequence in $U$, with limit $x$. For $\varepsilon>0$ there exists an integer $n_{0}$ such that $n>n_{0}$ implies 


$$
\begin{aligned}
d(x, f(x))< & \varepsilon+d\left(f\left(x_{n}\right), f(x)\right) \\
< & \varepsilon+a d\left(x_{n}, x\right)+b\left[d\left(x_{n}, f\left(x_{n}\right)\right)+d(x, f(x))\right] \\
& +c\left[d\left(x_{n}, f(x)\right)+d\left(x, f\left(x_{n}\right)\right)\right] \\
\leqslant & 2 \varepsilon+(b+c) d(x, f(x)) .
\end{aligned}
$$

Therefore $(1-b-c) d(x, f(x)) \leqslant 2 \varepsilon$ and $x=f(x)$.

Let $x_{1}, x_{2} \in U, x=\left(x_{1}+x_{2}\right) / 2$.

$$
\begin{aligned}
& d(x, f(x)) \leqslant \frac{1}{2}\left[d\left(f\left(x_{1}\right), f(x)\right)+d\left(f\left(x_{2}\right), f(x)\right)\right] \\
& \leqslant \frac{1}{2}\left[a_{1} d\left(x_{1}, x\right)+b_{1}\left\{d\left(x_{1}, f\left(x_{1}\right)\right)+d(x, f(x))\right\}\right. \\
& \quad+c_{1}\left\{d\left(x_{1}, f(x)\right)+d\left(x, f\left(x_{1}\right)\right)\right\}+a_{2} d\left(x_{2}, x\right) \\
& \quad+b_{2}\left\{d\left(x_{2}, f\left(x_{2}\right)\right)+d(x, f(x))\right\} \\
& \left.\quad+c_{2}\left\{d\left(x_{2}, f(x)\right)+d\left(x, f\left(x_{2}\right)\right)\right\}\right] .
\end{aligned}
$$

Therefore

$d(x, f(x))$

$$
<\frac{\left(a_{1}+c_{1}\right) d\left(x, x_{1}\right)+c_{1} d\left(x_{1}, f(x)\right)+\left(a_{2}+c_{2}\right) d\left(x, x_{2}\right)+c_{2} d\left(x_{2}, f(x)\right)}{2-b_{1}-b_{2}} .
$$

It can be shown that $d\left(x_{i}, f(x)\right) \leqslant d\left(x_{1}, x_{2}\right) / 2$, so that $d(x, f(x))<$ $d\left(x_{1}, x_{2}\right) / 2$.

But $d\left(x_{1}, x_{2}\right) \leqslant d\left(x_{1}, f(x)\right)+d\left(x_{2}, f(x)\right) \leqslant d\left(x_{1}, x_{2}\right)$. Since $X$ is strictly convex, $x_{i}-f(x)$, hence $f(x)$ must lie on the line segment joining $x_{1}$ and $x_{2}$. The inequalities $d\left(x_{i}, f(x)\right) \leqslant d\left(x_{1}, x_{2}\right) / 2$ imply that $f(x)$ is the midpoint. Therefore $x=f(x)$.

Theorem 3 of [28] is the special case of this theorem with $c=0$.

Replacing $f$ by $f^{p}$ in Theorems $26-28$ yields corresponding results for the mappings (46) and (34).

A theorem similar to Theorem 26, for a pair of continuous mappings satisfying (129) for $a=\frac{1}{2}$, appears in [30].

8. Conclusion. We conclude this paper by indicating, in the form of open questions, directions for further work.

1. If $f \in(25)$, is continuous on $X$, and $\left\{f^{n}\left(x_{0}\right)\right\}$ has a cluster point for some $x_{0} \in X$, does $f$ possess a fixed point?

2. If the answer to 1 is no, then what additional hypotheses on $f$ or $X$ are needed in (25) to guarantee the existence of a fixed point?

3. If $f$ satisfies any of the definitions (95)-(100), does $f$ have a fixed point? 
4. If $f$ satisfies any of the definitions (104)-(125), does $f$ have a fixed point?

5. Is the concept of locally contractive extendable to (11), (36), (61), (86), or (111)?

6. What are the answers to $1-4$ for the corresponding definitions for a pair of mappings $f, g$ ? a sequence of mappings?

ADDED IN PROOF. 1. L. Khazanchi [Math. Japon. 19 (1974), 283-289] has proved a fixed point theorem for functions satisfying (89) with $2(a+b)+c$ $<1$. The author, in a paper submitted for publication, has extended this result to functions satisfying (94).

2. Preprints of this paper were circulated prior to publication. Dr. M. Maiti has made the observation that, with respect to the definitions mentioned in Theorem $2, f$ need not be continuous over all of $X$. It is sufficient to require $f$, and some iterate of $f$, to be continuous at a cluster point of $\left\{f^{n}\left(x_{0}\right)\right\}$.

\section{REFERENCES}

1. D. F. Bailey, Some theorems on contractive mappings, J. London Math. Soc. 41 (1966), 101-106. MR 32 \#6434.

2. L. P. Belluce and W. A. Kirk, Fixed-point theorems for certain classes of nonexpansive mappings, Proc. Amer. Math. Soc. 20 (1969), 141-146. MR 38 \# 1663.

3. R. M. T. Bianchini, Su un problema di S. Reich riguardante la teoria dei punti fissi, Boll. Un. Mat. Ital. 5 (1972), 103-108.

4. D. W. Boyd and J. S. W. Wong, On nonlinear contractions, Proc. Amer. Math. Soc. 20 (1969), 458-464. MR 39 \#916.

5. S. K. Chatterjea, Fixed-point theorems, C. R. Acad. Bulgare Sci. 25 (1972), 727-730. MR 48 \#2845.

6. - Fixed point theorems for a sequence of mappings with contractive iterates, Publ. Inst. Math. (Beograd) 14 (28) (1972), $15-18$.

7. L. B. Ciric, Generalized contractions and fixed-point theorems, Publ. Inst. Math. (Beograd) (N.S.) 12 (26) (1971), 19-26. MR 46 \#8203.

8. A generalization of Banach's contraction principle, Proc. Amer. Math. Soc. 45 (1974), 267-273. MR 50 \#8484.

9. M. Edelstein, An extension of Banach's contraction principle, Proc. Amer. Math. Soc. 12 (1961), 7-10. MR 22 \#11375.

10. - On fixed and periodic points under contractive mappings, J. London Math. Soc. 37 (1962), 74-79. MR 24 \# A2936.

11. K. Goebel, W. A. Kirk and T. N. Shimi, A fixed point theorem in uniformly convex spaces, Boll. Un. Mat. Ital. (4) 7 (1973), 67-75. MR 47 \#9367.

12. V. K. Gupta and P. Srivastava, A note on common fixed points, Yokahama Math. J. 19 (1971), 91-95.

13. , On common fixed points, Rev. Roumaine Math. Pures Appl. 17 (1972), 531-538. MR 46 \#4287.

14. L. F. Guseman, Jr., Fixed point theorems for mappings with a contractive iterate at a point, Proc. Amer. Math. Soc. 26 (1970), 615-618. MR 42 \#19.

15. G. E. Hardy and T. D. Rogers, A generalization of a fixed point theorem of Reich, Canad. Math. Bull. 16 (1973), 201-206. MR 48 \#2847.

16. K. Iseki, On common fixed points of mappings, Bull. Austral. Math. Soc. 10 (1974), 365-370. MR 50 \#8487.

17. R. Kannan, Some results on fixed points, Bull. Calcutta Math. Soc. 60 (1968), 71-76. MR $41 \# 2486$. 
18. - Some results on fixed points. II, Amer. Math. Monthly 76 (1969), 405-408. MR 41 \#2487.

19. S. Massa, Un'osservazione su un teorema di Browder-Roux-Soardi, Boll. Un. Mat. Ital. (4) 7 (1973), 151-155. MR 47 \#4080.

20. N. Muresan, Familii de applicatii si puncte fixe (Families of mappings and fixed points), Studia Univ. Babes-Bolyai Ser. Math.-Mech. 19 (1974), 13-15. MR 49 \#6214.

21. E. Rakotch, A note on contractive mappings, Proc. Amer. Math. Soc. 13 (1962), 459-465. MR 26 \#5555.

22. B. Ray, Some results on fixed points and their continuity, Colloq. Math. 27 (1973), 41-48. MR 49 \#1501.

23. B. K. Ray, On non-expansive mappings in a metric space, Nanta Math. 7 (1974), 86-92.

24. S. Reich, Some remarks concerning contraction mappings, Canad. Math. Bull. 14 (1971), 121-124. MR 45 \#1145.

25. - Kannan's fixed point theorem, Boll. Un. Mat. Ital. (4) 4 (1971), 1-11. MR 46 \#4293.

26. - Fixed points of contractive functions, Boll. Un. Mat. Ital. (4) S (1972), 26-42. MR 46 \#8206.

27. - Remarks on fixed points, Atti. Accad. Naz. Lincei Rend. Cl. Sci. Fis. Mat. Natur. (8) 52 (1972), 689-697. MR 48 \#9473.

28. D. Roux and P. Soardi, Alcune generalizzazioni del teorema di Browder-Gobhde-Kirk, Atti Accad. Naz. Lincei Rend. Cl. Sci. Fix. Mat. Natur. (8) 52 (1972), 682-688. MR 48 \#4856.

29. I. A. Rus, On common fixed points, Studia Univ. Babę-Bolyai Ser. Math.-Mech. 18 (1973), 31-33. MR 49 \# 1502.

30. M. Seelbach, Some common fixed point theorems for compact metric spaces, Notices Amer. Math. Soc. 21 (1974), A-179. Abstract \#711-46-23.

31. V. M. Sehgal, On fixed and periodic points for a class of mappings, J. London Math. Soc. (2) 5 (1972), 571-576. MR 47 \# 7722.

32. S. P. Singh, Some results on fixed point theorems, Yokahama Math. J. 17 (1969), 61-64. MR 41 \#2488.

33. P. Soardi, Su un problema di punto unito di S. Reich, Boll. Un. Mat. Ital. (4) 4 (1971), 841-845. MR 46 \# 741.

34. C. S. Wong, Common fixed points of two mappings, Pacific J. Math. 48 (1973), 299-312. MR 48 \#7245.

35. C.-L. Yen, Remark on common fixed points, Tamkang J. Math. 3 (1972), 95-96. MR 48 \# 1206.

36. T. Zamfirescu, $A$ theorem on fixed points, Atti Acad. Naz. Lincei Rend. Cl. Sci. Fis. Mat. Natur. (8) 52 (1972), 832-834 (1973). MR 48 \#246.

37. - Fix point theorems in metric spaces, Arch. Math. (Basel) 23 (1972), 292-298. MR 46 \#9957.

Department of Mathematics, Indina University, Bloominoton, Indiana 47401 